\title{
The mid-developmental transition and the evolution of animal body plans
}

\author{
Michal Levin ${ }^{\# 1,+}$, Leon Anavy ${ }^{\# 1}$, Alison G. Cole ${ }^{1}$, Eitan Winter ${ }^{1}$, Natalia Mostov ${ }^{1}$, Sally \\ Khair $^{1}$, Naftalie Senderovich ${ }^{1}$, Ekaterina Kovalev ${ }^{1}$, David H. Silver ${ }^{1}$, Martin Feder ${ }^{1}$, Selene \\ L. Fernandez-Valverde ${ }^{2, \#}$, Nagayasu Nakanishi ${ }^{2, \%}$, David Simmons ${ }^{3}$, Oleg Simakov ${ }^{4}$, Tomas \\ Larsson ${ }^{4}$, Shang-Yun Liu ${ }^{5}$, Ayelet Jerafi-Vider ${ }^{6}$, Karina Yaniv $^{6}$, Joseph F. Ryan ${ }^{3}$, Mark Q. \\ Martindale $^{3}$, Jochen C. Rink ${ }^{5}$, Detlev Arendt ${ }^{4}$, Sandie M. Degnan ${ }^{2}$, Bernard M. Degnan², \\ Tamar Hashimshony ${ }^{1}$, and Itai Yanai ${ }^{1, \&}$ \\ ${ }^{1}$ Department of Biology, Technion - Israel Institute of Technion, Haifa, Israel \\ ${ }^{2}$ School of Biological Sciences, University of Queensland, Brisbane, QLD, Australia \\ ${ }^{3}$ Whitney Laboratory for Marine Bioscience, University of Florida, $9505 \mathrm{~N}$, Ocean Shore Blvd, St. \\ Augustine, FL 32080-8610 USA \\ ${ }^{4}$ Developmental Biology Unit, European Molecular Biology Laboratory, Heidelberg, Germany \\ ${ }^{5}$ Max Planck Institute of Molecular Cell Biology and Genetics, Pfotenhauerstrasse 108, 01307 \\ Dresden, Germany \\ ${ }^{6}$ Department of Biological Regulation, Weizmann Institute of Science, Rehovot, Israel \\ \# These authors contributed equally to this work.
}

\section{Abstract}

Animals are grouped into $\sim 35$ 'phyla' based upon the notion of distinct body plans ${ }^{1-4}$.

Morphological and molecular analyses have revealed that a stage the middle of developmentknown as the phylotypic period-is conserved among species within some phyla ${ }^{5-9}$. While these analyses provide evidence for their existence, phyla have also been criticized as lacking an objective definition, and consequently based on arbitrary groupings of animals ${ }^{10}$. Here, we compare the developmental transcriptomes of ten species, each annotated to a different phylum, with a wide range of life histories and embryonic forms. We find that, in all ten species,

Users may view, print, copy, and download text and data-mine the content in such documents, for the purposes of academic research, subject always to the full Conditions of use:http://www.nature.com/authors/editorial_policies/license.html\#terms

${ }^{\&}$ Correspondence and requests for materials should be addressed to I.Y. yanai@ technion.ac.il.

\#Present address: Laboratorio Nacional de Genómica para la Biodiversidad, Centro de Investigación y de Estudios Avanzados del IPN, Irapuato, Guanajuato, Mexico.

${ }^{+}$Present address: Center for Thrombosis and Hemostasis (CTH), University Medical Center Mainz, Mainz, Germany.

\% Present address: Whitney Laboratory for Marine Bioscience, University of Florida, 9505 N, Ocean Shore Blvd, St. Augustine, FL 32080-8610 USA.

Supplementary Information:

This submission includes seven extended data figures and three extended data tables and one supplementary table.

Author information:

The complete data set has been deposited to the NCBI GEO database GSE70185.

The authors declare no competing financial interests. 
development comprises the coupling of early and late phases of gene expression. These conserved phases are linked by a divergent 'mid-developmental transition' that deploys species-specific suites of signaling pathways and transcription factors. This mid-developmental transition overlaps with the phylotypic period that has been defined previously for three of the ten phyla, suggesting that transcriptional circuits and signaling mechanisms active during this transition are crucial for defining the phyletic body plan and that the mid-developmental transition may be used to define phylotypic periods in other phyla. Placing these observations alongside the reported conservation of mid-development within phyla, we propose that a phylum may be defined as a collection of species whose gene expression at the mid-developmental transition is both highly-conserved among them, yet divergent relative to species in other phyla.

To study the broad patterns of embryonic development, we selected ten distantly-related species that collectively provide a wide sampling of the variation exhibited by the animal kingdom at the level of morphological and developmental complexity (Figure 1 and Extended Data Table 1). This collection includes a single species from poriferans, cnidarians, nematodes, arthropods, chordates, echinoderms, annelids, platyhelminthes, ctenophores, and tardigrades. Seven of the species are bilaterians-five protostomes and two deuterostomes - while the cnidarian Nematostella vectensis represents a clade that is the sister group to bilaterians, and the ctenophore Mnemiopsis leidyi and the poriferan Amphimedon queenslandica represent two earlier branching taxa ${ }^{11}$. Collectively, these species provide a unique platform for the study of global features in animal development.

For each species, we isolated on average 70 individual embryos spanning development (Figure 1b). The transcriptome of each embryo was analyzed using CEL-Seq ${ }^{12}$. For three species-Hypsibius dujardini (tardigrade), Schmidtea polychroa (platyhelminth), and Platynereis dumerilii (annelid) - where a published genome was unavailable, we first produced a comprehensive developmental transcriptome (Extended Data Figure 1, Extended Data Tables 2, 3, and Supplementary Table 1). To assay dynamic expression such that it is not overly biased by individual embryos, for each of the ten time-courses we computed expression across twenty overlapping sliding windows of the embryos, sorted by BLIND ${ }^{13}$ (Extended Data Figure 2a), and used these averaged expression profiles in our analyses.

Figure 2 shows the standardized expression profiles of the dynamically expressed genes across the ten species (Extended Data Figure 2b). To compare gene expression across these species, we delineated 11,139 orthologous protein families, with each orthologous family having representatives from an average of six species. The expression of eleven $C$. elegans transcription factors-conserved across the other species in this study-are indicated in Figure 2, highlighting how this dataset can serve as an important resource for developmental and evolutionary biologists. For example, the TCF/LEF family is expressed in the early phases of six of the time-courses and later in the remaining species (Figure 2, dark squares, named pop-1 in C. elegans).

To systematically compare gene expression across species, we computed the correlation across orthologous gene expression throughout development for each pair of species. For example, comparison of tardigrade and annelid embryonic transcriptomes strikingly revealed two conserved phases of expression in these two species - early and late - separated by a 
sharp mid-developmental transition $\left(P<10^{-10}\right.$, Kolmogorov-Smirnov test, Figure 3, inset; Extended Data Figure 3). These observations indicate that although the external embryonic development of these two species is enormously different, at the level of orthologous gene expression their early and late development is comparable. Moreover, while development appears morphologically gradual in both species, it is punctuated by a dramatic change in gene expression during mid-development. Comparing all pairs of species extended this result, even to the ctenophore and the sponge, suggesting that this dual-phase feature of development dates back to the common ancestor of all animals (Figure 3). The broad extent of this behavior is remarkable, especially considering the challenges associated with the de novo assembly of transcriptomes and reliance upon inferred gene models. Deviations from the dual-phase pattern may occur for biological reasons. For example, the early embryos of the planarian S. polychroa contain substantial amounts of maternal RNA, which appears as a third initial phase in all comparisons with that species. Other deviations appear more cryptic, such as the correlation matrix between the cnidarian and the sponge. Overall, the dual-phase pattern holds for most pairwise species comparisons, with the exception of nine out of 45 (Extended Data Figure 3), and is robust to the parameters used for constructing the sliding window expression profiles and to possible biases in the embryo sampling (Extended Data Figure 4ab).

To study the deeply conserved expression modules we asked which Gene Ontology functional categories are enriched in the genes occurring in each of the two phases. Focusing on the stages spanning three windows prior to and following the transition, we identified genes whose expression is restricted to the early and late phases (Extended Data Figure 5). We found that orthologous groups of genes that tend to be expressed in the early phase across the ten species are enriched for chromatin changes, cell cycle, and the regulation of gene expression (Extended Data Figure 5), suggesting that this phase is characterized by the expression of genes involved in the cell biology of proliferation. The late phase, in contrast, was enriched with cell-type specific genes such as various transporters, metabolic enzymes, and synaptic transmission factors that together reflect a period of differentiation.

We next asked if the transition between the early and late phases is enriched in the expression of genes of particular functional groups, and found significant enrichments for signaling processes such as Notch and Wnt during this transition (Extended Data Figure 5). Thus, a common signature among the animals studied here is that, between periods of cell proliferation and differentiation, a period of intense signaling occurs, which is expected from a developmental biology perspective ${ }^{14}$. To study the expressed signaling pathways in greater detail, we examined seven major pathways and tested for their enrichment during the transition between the two phases in each of the ten species (Figure 4a). We found a pattern of variation indicating that each of the distantly related species deploys a distinct suite of signaling pathways during the transition. Extending this analysis to transcription factor families, we found that only the homeobox gene family is enriched at the middevelopmental transition in all ten species, as might be expected; otherwise, each species expresses a unique combination of the major developmental transcription factors (Figure $4 b)$. A distinct combination of signaling pathways and transcription factors during the middevelopmental transition may thus be of particular importance to the development of specific body plans. 
Mapping the timing of these mid-developmental transitions back onto the embryonic timecourse of nematodes, arthropods, and chordates we found that they overlap—or partially overlap — with the previously described phylotypic periods of these animals (Figure 1b). Specifically, in nematodes the transition maps to the end of the ventral enclosure stage which has been proposed as the phylotypic period ${ }^{7}$ (Figure 1b). In chordates, the transition maps to the early part of the pharyngula stage, which for this group of animals has been assigned the phylotypic period $^{9,15}$. Furthermore, in arthropods, the transition centers upon the head involution and dorsal closure stage but also begins at the end of the germ band stage, which has been assigned as the phylotypic period in this phylum ${ }^{16,17}$. Given this fair correspondence, we propose that the mid-developmental transition uncovered in this study marks a phylum's phylotypic period. We note, however, that the lack of complete correspondence of the arthropod mid-developmental transition with the germ-band stage will require further analysis and that this provisional definition will also need refinement to account for other taxonomic ranks (e.g. Class) and for the diversity of life cycles within a phylum.

In the annelid, the mid-developmental transition corresponds to the late trochophore stage, which overlaps the onset of differentiation of the first three larval segments, stomodeal opening (mouth), and ventral nerve cord ${ }^{18}$. These features have been proposed to define the phylotypic period in annelids ${ }^{19}$. Interestingly, the trochophore larva is common to other spiralian phyla (e.g. molluscs and nemerteans). In the flatworm, which undergoes a highly derived mode of development ${ }^{20}$, the mid-developmental transition corresponds to the stage in which the embryonic pharynx is joined by a second 'adult-pharynx', consistent with the phylotypic period previously proposed for this phylum ${ }^{21}$. In the sponge, the middevelopmental transition may occur between the cloud and spot stages, when the primary larval axis is established ${ }^{22}$. We note however that in species with more complex life cycles with several phases of differentiation a single mid-developmental transition may be less accentuated.

Finally, we measured the extent of evolutionary change within the two conserved phases and the mid-developmental transition by determining whether orthologs annotated for a particular temporal category in one species are also annotated to the same temporal category in another species. Figure $4 \mathrm{c}$ shows an example of this analysis for $D$. melanogaster and $C$. elegans. For 4,395 orthologs delineated between these two species, the early phase, middevelopmental transition, and the late phase expression account for $51 \%, 14 \%$, and $35 \%$ of the $C$. elegans orthologs, respectively. $28 \%$ of the orthologs are annotated to the early phase in both $C$. elegans and D. melanogaster, while by chance only $22 \%$ are expected given the fraction of genes in each category across the species (Figure $4 \mathrm{c}$ ). In contrast, $3 \%$ were expected to be conserved at the mid-developmental transition at random, and 3\% were observed. The log-odds ratios between observed and expected for the early phase and the mid-developmental transition between C. elegans and D. melanogaster are thus 0.35 and 0 , respectively. Comparing the log-odds ratios across the three temporal categories for each of the 45 pairs of the ten species, we found that the mid-developmental transition profiles are significantly less conserved than the early and late phase expression (Figure $4 \mathrm{~d}, P<10^{-6}$ compared with the early phase and $P<10^{-12}$ with the late phase, Kolmogorov-Smirnov test). 
We found a similar result when comparing at the level of PFAM domains (Extended Data Figure 6,7).

Our results are consistent with an inverse hourglass model for metazoan body plans (Figure 4 e), where the molecular components that comprise early and late embryogenesis are more conserved, and the signaling pathways and transcription factors acting within the middevelopmental transition are variable across major animal lineages (Figure 4ab). Interestingly, this is inverse to the model summarizing comparisons made within a phylum, where gene expression differences across species are minimal at the phylotypic period ${ }^{23}$. Consequently, a 'phylum' may be defined as a set of species sharing the same signals and transcription factor networks during the mid-developmental transition. As a result, transcriptional variance will have an hourglass-shape within the phylum, and the inverse when comparing species across phyla (Figure 4e). A non-phylum lower taxon would not meet these criteria since an hourglass pattern of similarities would be observed both within the taxon and across more distant species. Should this transcriptomic definition hold, evidence will be provided for the usefulness of 'phylum' as a biological classification. It may also suggest the delineation of new phyla, as well as the collapsing of previously distinct ones, requiring validation by zoological studies. We note that the topology of an inverted hourglass has been previously proposed for heterochrony at the phylotypic period ${ }^{24}$, though it was not invoked in the context of inter-phylum divergence.

While the Hox cluster has been implicated with the metazoan 'zootype' ${ }^{\text {,25 }}$, we find that even organisms without Hox genes (Amphimedon ${ }^{26}$ and Mnemiopsis $^{27}$ ) have a middevelopmental transition period at a time consistent with a phylotypic period (Figure 1b). Thus, more ancient than the patterning of the body axis by Hox genes, may be the molecular constraints of the transition from a general phase of proliferation to a phase of signaling and differentiation that results in the positioning of cells in a phylum-specific manner. Such a transition may be a hallmark of development only in animals, or future work may show that this is a general characteristic of development in all multicellular life. It will be interesting to further employ systematic analyses in studying the developmental constraints on metazoans and other clades 8,28 .

\section{METHODS}

\section{Sample collection}

Platynereis dumerilii embryos were collected in EMBL Heidelberg, Germany. In each of several containers, a gravid male and a female were mixed in a small container containing North Sea water. The classical breeding dance was observed after several minutes and the females and males released oocytes and sperm. Fertilized eggs were incubated at $18^{\circ} \mathrm{C}$ and embryos were collected every hour after fertilization for a period of 5 days. Individual embryos were collected on the cap of a $1.5 \mathrm{ml}$ Eppendorf tube using a micro-mouth pipette. Excessive water was removed and the sample was flash-frozen in liquid nitrogen.

Schmidtea polychroa embryos were collected at the Max Planck Institute CBG, Dresden, Germany. A population of $S$. polychroa was maintained in the lab at $20^{\circ} \mathrm{C}$ as previously described $^{32}$. Egg capsules were regularly collected over 15 days of development just after 
deposition and kept in Petri dishes at $20^{\circ} \mathrm{C}$. To release embryos for isolation, capsules were carefully opened using two fine forceps. After assessment of stage of development according to the Martin-Duran system ${ }^{33}$ excess water was removed and embryos were flash-frozen in Eppendorf tubes in liquid nitrogen.

Hypsibius dujardini starting cultures were provided by Bob Goldstein (University of North Carolina at Chapel Hill) and embryos were collected as previously described ${ }^{34}$ at the Technion, Israel. Small cultures of tardigrades were kept in $60 \mathrm{~mm}$ glass Petri dishes in commercial bottled spring water until gravid animals were visible. Tardigrades lay 2-5 eggs during molting, with the embryos deposited in their shed exoskeleton, the exuvia. Soon after the adult crawled out of the exuvia, it was cut open using a scalpel on a microscope-cavityslide to release embryos into the medium. Embryos were observed using a standard binocular and when reaching two-cell stage were deposited in a $10 \mu \mathrm{l}$ drop mineral water on the cap of a $1.5 \mathrm{ml}$ Eppendorf tube. Tubes were closed and incubated for respective periods at $20^{\circ} \mathrm{C}$. For 4.5 days every hour past two-cell stage embryos were inspected for viability, excessive water was removed using a micro-mouth pipette, and the tube was flash-frozen in liquid nitrogen.

Drosophila melanogaster embryos were collected at the Technion using a previously published protocol ${ }^{35}$. Briefly, agar plates with apple juice smeared with freshly prepared yeast were used to make young adult flies lay a lot of eggs. Cages consisting of such plates were set up with at least 20 flies and left for roughly one day for the flies to acclimatize. Plates were replaced with fresh ones twice in one hour interval to ensure use only newly laid eggs. Drosophila embryos are covered with a non-transparent chorion which has to be removed prior to live imaging by dechorionation. Shortly after being laid, embryos were washed off from plates into a plastic sieve using tap water and a fine brush to loosen the embryos. In the sieve, embryos were submerged in 50\% bleach solution for two minutes. Embryos were washed and rinsed with cold water. Using a needle pick, 20 embryos were placed in a row on a strip of agar placed on a glass slide. $\mathrm{n}$-Heptane glue was applied in a thin layer on a big glass cover slip. This cover slip was carefully put upside down on top of the embryos on the agar strip so that embryos adhere to the glue layer. Embryos were covered with PBS and kept in humid chambers at $25^{\circ} \mathrm{C}$. Embryos on the slip were observed under light microscope and for each embryo, the time of cellularization was noted. To collect an embryo, a needle pick was used to carefully remove it from the slide and it was flash-frozen in an Eppendorf tube in liquid nitrogen.

Strongylocentrotus purpuratus oocytes and sperm were kindly provided by Smadar Ben Tabou deLeon (Haifa University, Israel) and mixed and cultured at the Technion. Mixing occurred by 4 drops of sperm in $50 \mathrm{ml}$ of eggs in sea water and incubated at $18^{\circ} \mathrm{C}$ in petri dishes. Every 40 minutes after fertilization for a period of 72 hours single embryos were deposited on the cap of a $1.5 \mathrm{ml}$ Eppendorf tube. Excessive water was removed using a micro-mouthpipette and the embryo was flash-frozen in liquid nitrogen.

Danio rerio fertilization was performed in the lab of Karina Yaniv (Weizmann Institute, Israel). Four female and one male Danio rerio fish were mixed in a breeder tank. Fertilized eggs were collected into zebrafish embryo medium as previously described ${ }^{36}$. Fertilized 
eggs were sampled in a small volume of medium every 40 minutes from fertilization into the cap of a $1.5 \mathrm{ml}$ Eppendorf tube. Excess water was removed using a micro mouth pipette and the embryo flash-frozen in liquid nitrogen.

Nematostella vectensis egg masses and sperm were provided by Amos Schaffer (Gat Lab, Hebrew University of Jerusalem). Eggs and sperms were mixed and egg jelly was dissolved as previously described ${ }^{37}$ using $4 \%$ cysteine ( $\mathrm{pH} 7.4-7.6$ ) to make single embryos accessible for collection. The embryos were washed of cysteine six times using $30 \%$ of sea water. Fertilized embryos were observed under light microscope and embryos reaching 4-cell stage were deposited in a 10ul drop of $30 \%$ salt water on the cap of a $1.5 \mathrm{ml}$ Eppendorf tube. Tubes were closed and incubated for respective periods at $20^{\circ} \mathrm{C}$. At collection time, embryos were inspected for viability and excessive water was removed using a micro mouth pipette. The embryo was then flash-frozen in liquid nitrogen. Stages ranged from 4-cell stage and every 20 minutes for up to 48 hours when embryos reached the late planula stage.

Mnemiopsis leidyi embryos were collected in the Whitney Institute, University of Florida as previously described ${ }^{38}$. Stages ranged from the fertilized egg to 20 hours. Three replicate time-courses each comprising 20 embryos were isolated. In one replicate, embryos were flash frozen and shipped on dry ice. In the other two RNA was prepared by a TRIzol extraction and shipped in $75 \%$ ethanol on dry ice.

\section{RNA-Seq transcriptome sequencing}

For Hypsibius dujardini, Schmidtea polychroa, and Platynereis dumerilii, RNA was isolated from a mixed population of embryos, larvae, and adults according to the TRIzol protocol (Invitrogen). This RNA was processed according to the Illumina TruSeq RNA-Seq protocol by the Technion Genome Center and 100bp paired-end sequencing was performed. To preprocess the reads, 'Sickle' ${ }^{39}$ was used for quality trimming with a threshold of 31 and Illumina adaptors were removed using 'Scythe' ${ }^{40}$. Sequencing error correction was next made using the AllpathLG toolkit ${ }^{41}$ and poly-A sequences were trimmed using trimest (Gary Williams, unpublished). The resulting libraries were cleaned of short and duplicate reads using the fastx toolkit (Assaf Gordon, unpublished). Hypsibius dujardini's genome has been recently reported by two groups ${ }^{42,43}$.

\section{Mapping to S. purpuratus and $\mathbf{N}$. vectensis}

The sea urchin transcriptome was downloaded from Echinobase - NCBI BioProject PRJNA81157. The longest Isoform per transcript was selected leaving 21,000 peptides. For this organism the mapping was done more loosely with bowtie parameters set to "--mp 3,1 N 1 -L 15" as the RNA-Seq was done on a heterogenic population. For Nematostella we retrieved the T1 transcriptome from Stellabase ${ }^{44}$. Using Transdecoder (https:// transdecoder.github.io) revealed that, of the $115 \mathrm{k}$ transcripts, only $\sim 53 \mathrm{k}$ encoded proteins. BLAST analysis of the encoded protein resulted in $\sim 42 \mathrm{~K}$ unique proteins and the longest transcript was selected for each protein. 


\section{CEL-Seq transcriptome sequencing}

Total RNA was extracted from single embryos using TRIzol as previously described ${ }^{7}$ including minor adjustments. After the addition of TRIzol to the embryos the mixture was frozen in liquid nitrogen, thawed at 37 degrees and vortexed for 30 seconds. This procedure was repeated five times. Chloroform was then added and the sample further processed. The dried total RNA pellet was dissolved in RNase free water before introduction into subsequent amplification and sequencing library preparation steps. Using the CEL-Seq protocol $^{45}, 1 \mu$ l of a single embryo total RNA sample with a maximum concentration of 50 $\mathrm{ng} / \mu \mathrm{l}$, was mixed with $1 \mu \mathrm{L}$ of the ERCC spike-in kit diluted according to the manufacturer's protocol ${ }^{46}$. The libraries were sequenced using Illumina paired-end sequencing as previously reported in the CEL-Seq protocol ${ }^{45}$. For Read 1, used to determine the barcode, the first $15 \mathrm{bp}$ were sequenced and for Read 2, used to determine the identity of the transcript, the first 35bp were sequenced. The CEL-Seq pipeline is available at GitHub.com.

\section{CEL-Seq initial analysis pipeline}

Transcript abundances were obtained from the sequencing data using custom scripts organized into a multistep paralleled computational pipeline. Briefly, after trimming and filtering, the paired-end reads were demultiplexed based on the first eight bases of the first read. For each sample, reads were mapped to a reference genome or transcriptome using bowtie 2 version $2.2 .3^{47}$ with default parameters and counted using htseq-count ${ }^{48}$ to generate read counts. Samples were filtered to include only samples with at least 500,000 reads and in additions ERCC spike-in information was also used to filter out samples with low correlation coefficients $(<0.65)$ to the known concentration or with high $(>0.3)$ spike-in to gene read count ratio. Read counts were then normalized by dividing by the total number of counted reads and multiplying by $10^{6}$. Because CEL-Seq retains only the 3' end of the transcript, this procedure yields the estimated gene expression levels in transcripts per million (tpm) without transcript length normalization. In this work, we compare the tpm developmental profiles for different genes and across orthologs, and such comparisons are generally robust to overall RNA content changes.

\section{De novo transcriptome assembly with stranding and 3' anchoring}

A de novo transcriptome was generated for S. polychroa, P. dumerilii and H. dujardinii. Since we had at our disposal CEL-Seq reads, in addition to the RNA-Seq reads, our strategy was to exploit the stranded and 3'-biased nature of CEL-Seq. The Trinity software ${ }^{49}$ was used to generate, for each of the three species, two de novo transcriptome assemblies: 1. Single-end CEL-Seq reads were used to generate a 3' biased stranded transcriptome, and 2. The CEL-Seq reads were combined with paired-end RNA-Seq reads were used to generate a combined transcriptome. For the CEL-Seq 3' assembly, we ran Trinity using the single-end mode with 'SS_lib_type' parameter set to 'F'. For the combined assembly we ran Trinity using the paired-end mode with default parameters. The two resulting transcriptomes were then used to generate a single 3' anchored stranded transcriptome. For each transcript (contig) in the first set, we identified the corresponding transcripts in the second set using BLAST $^{50}$. Of those identified, we selected the transcript with the highest alignment score and used the strand information of the transcript in the first set to generate a stranded 
transcript (Extended Data Figure 1). Genes with alternative 3'-ends may be represented as distinct genes in this set, in those rare cases when the CEL-Seq contigs do not overlap. The generated set of transcripts was further filtered to contain only transcripts with a predicted protein using the Trinotate pipeline that is a part of the Trinity software ${ }^{49}$. PFAM domains ${ }^{51}$ were then identified using HMMER $^{52}$.

\section{Gene ontology and PFAM}

GO annotations for each transcriptome were generated using Trinotate (http:// trinotate.github.io/). Specifically, transcripts were searched against Uniprot sequences (comprising SwissProt and Trembl invertebrate, vertebrate, mammal, rodents and human data, clustered to $90 \%$ identity). GO and PFAM identifiers were then extracted from Uniprot accessions.

\section{Delineation of orthologous clusters}

OrthoMCL ${ }^{53}$ was used to delineate orthologous clusters from the ten proteomes of the ten species using the following parameters: "percentMatchCutoff" was set to 24, "evalueExponentCutoff" was set to -5, and the MCL parameters were "--abc -I 1.5". In the case of multiple genes in an orthology cluster for a particular species, the one with the highest fold-change was selected as the representative. We found similar results if the representative is selected randomly among the inparalogs.

\section{Developmental gene expression profiles}

Each time-course was initially ordered using BLIND - an automated method for determining the developmental order of transcriptomic samples ${ }^{13}$ (Extended Data Figure 2a). These profiles were smoothed using a moving average calculation with span parameter set to 3. In order to compare profiles of equal lengths, for each species we reduced the timecourse to twenty sliding windows using the following method. We defined the size of the window such that there is only overlap between every two consecutive windows. For each window the average expression was calculated for each gene across the included embryos. For each time-course, dynamic genes were defined as those with minimum expression of 10 transcripts per million and at least a two-fold change. Standardized expression was used in analysis where noted: to generate a standardized expression, the mean expression value was subtracted from each expression value and the results were divided by the standard deviation. To generate the phasegrams shown in Figure 2 we first standardized the $\log _{10}$ profiles by subtracting the mean and dividing by the standard deviation. We next computed the first two principal components of this expression data; since the profiles were standardized, the genes form a circle. The genes are then sorted according to their angle from the origin in this space. A gene expression profile was mapped to a temporal phase (early, transition, or late) by computing the correlation with the three idealized profiles shown in Extended Data Figure 5 and assigning it to the pattern exhibiting the highest correlation and thus best match. 


\section{Mid-developmental transition detection}

The transition period for each species was computed based upon the transcriptome similarities with the transcriptomes of the other species, shown in Figure 3. The twenty transcriptomes were clustered using hierarchical clustering based upon the Euclidean distances among their profiles of correlations with the profiles of all other species. The two deepest clusters were then identified and the precise temporal window separating them was set as the mid-developmental transition period.

\section{Gene Ontology (GO) enrichment analysis}

A temporal phase was assigned to each orthologous group by annotating it to its most represented phase. The $C$. elegans Gene ontology annotation was used on the $C$. elegans orthologs. Enrichment was computed using the hypergeometric distribution. In order to avoid retrieving enrichments due to the same set of genes we carried out serial enrichments as follows. The most enriched gene ontology group was noted, its genes removed from the set, and enrichment search was repeated to detect additional Gene Ontology terms. For the signaling pathways shown in Figure $4 \mathrm{~b}$ the following gene ontology terms were used: 'Wnt signaling pathway', 'Notch signaling pathway', 'hedgehog receptor activity', 'epidermal growth factor receptor signaling pathway', 'transforming growth factor beta receptor signaling pathway', 'MAPK cascade', 'G-protein coupled receptor activity'. For this analysis, we searched for enrichment up to three windows before and after the inferred transition, and kept the most significant $P$-value for each pathway (Hypergeometric distribution).

\section{PFAM signatures}

For each of 5,745 PFAMs, we computed an enrichment profile throughout time, and for each species, as follows. For each of the twenty expression windows of the matrix of standardized $\log _{10}$ expression values of the dynamic genes, we marked genes with expression above 0.5 as expressed. We then calculated the fraction of the genes within this set that contain genes annotated to the PFAM domain. A temporal phase was annotated using supervised clustering using the same approach shown in Extended Data Figure 5. For the transcription factor families shown in Figure 4d the following PFAMs were used: 'Homeobox domain', 'GATA zinc finger', 'Ligand-binding domain of nuclear hormone receptor', 'Helix-loop-helix DNAbinding domain', 'bZIP transcription factor', 'Zinc finger, C4 type (two domains)', 'Zinc finger, $\mathrm{C} 2 \mathrm{H} 2$ type', and 'T-box'. For this analysis, we searched for enrichment up to three windows before and after the inferred transition, and kept the most significant $P$-value for each TF family (Hypergeometric distribution). 


\section{Extended Data}

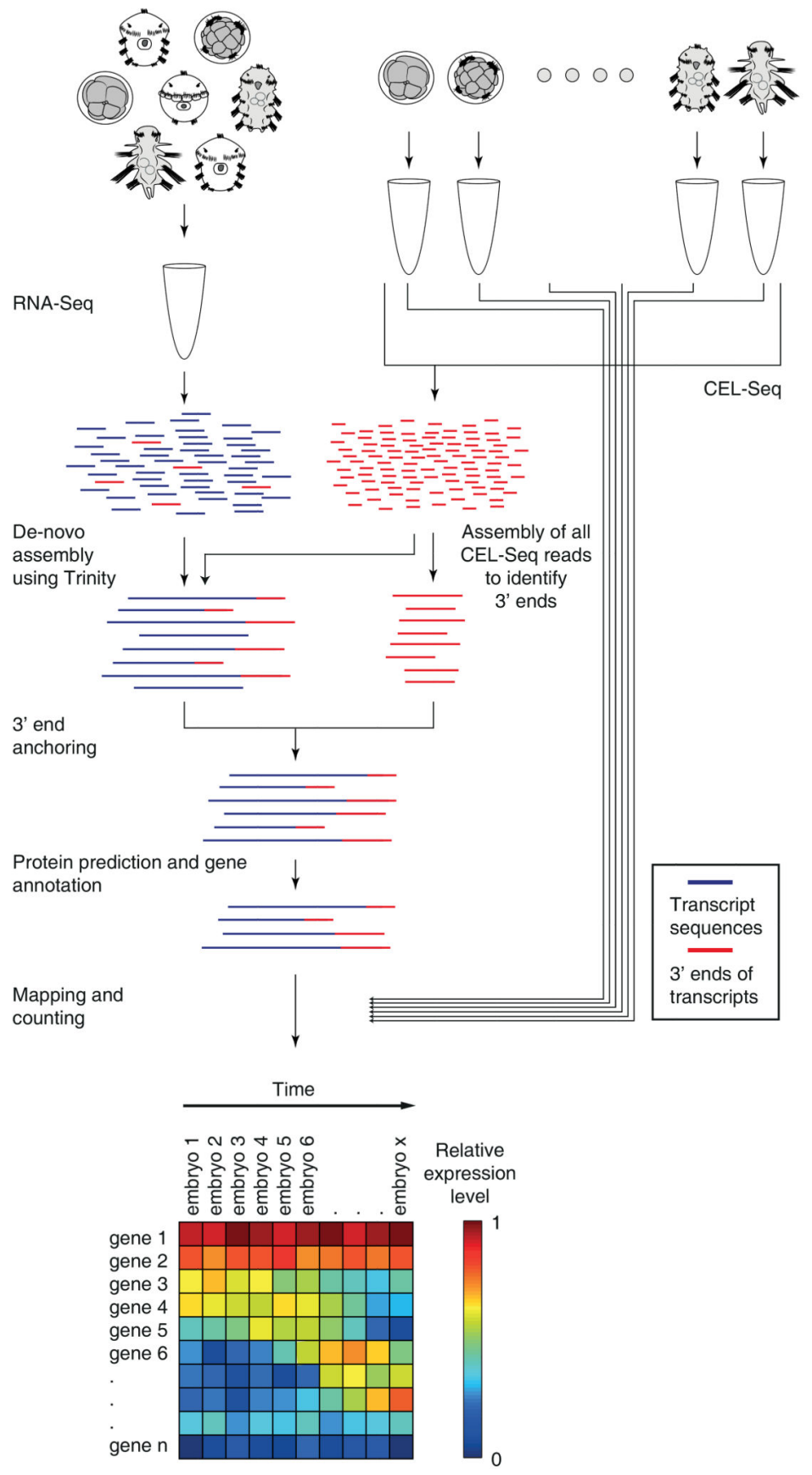

Extended Data Figure 1.

A schematic for the de novo transcriptome analysis (see also Methods). CEL-Seq reads were mapped to the published transcriptomes where available. 


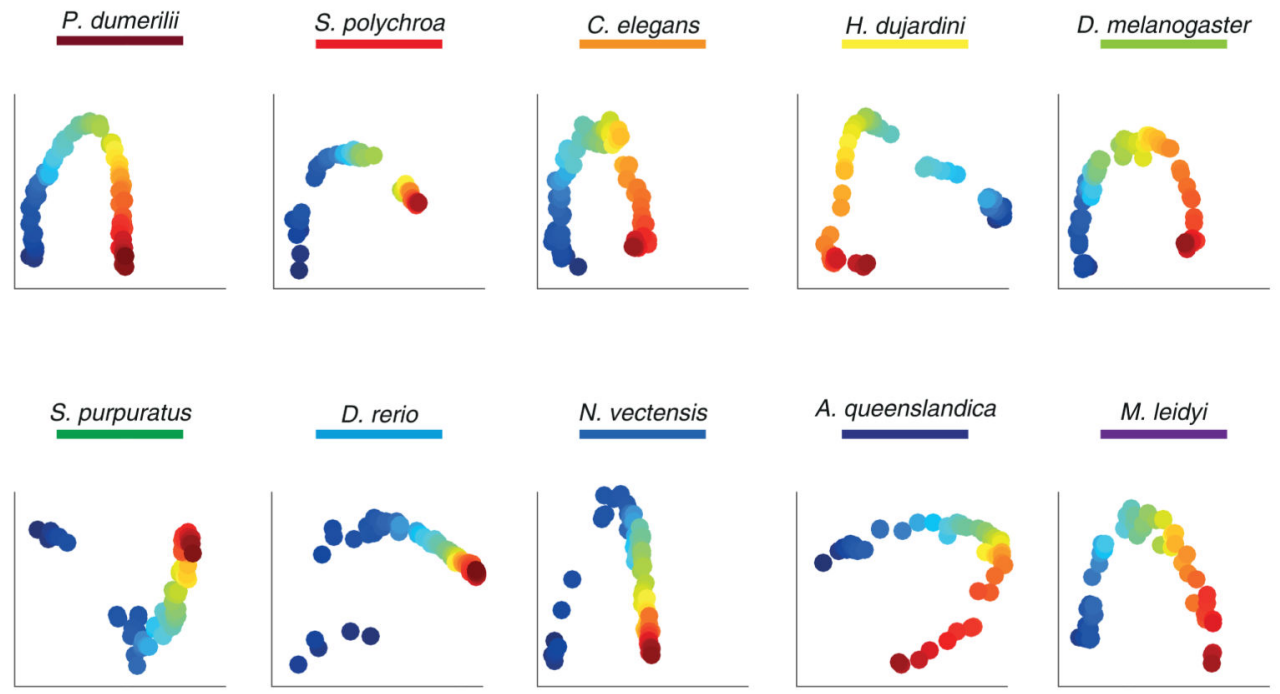

b

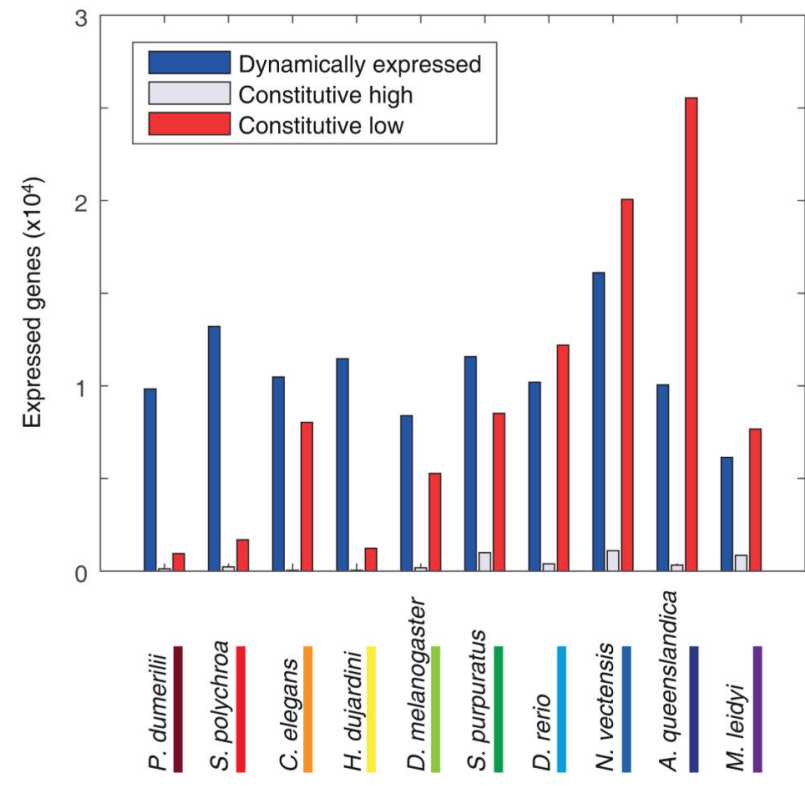

Extended Data Figure 2.

a. BLIND analysis on the reported time-courses. The color indicates the ordering. The species is indicated for each plot. b. The number of dynamically expressed genes for each species. The species are shown in the same order as in the main figures. Constitutively high (low) expression is defined as that where the maximum expression is more (less) than the 10 tpm threshold yet is not dynamic (two-fold change and at least 10 transcripts per million maximum expression). 
a

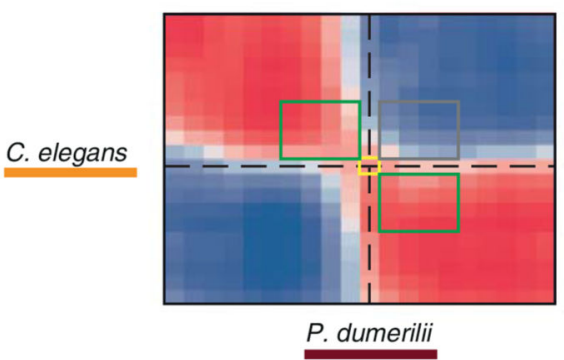

b

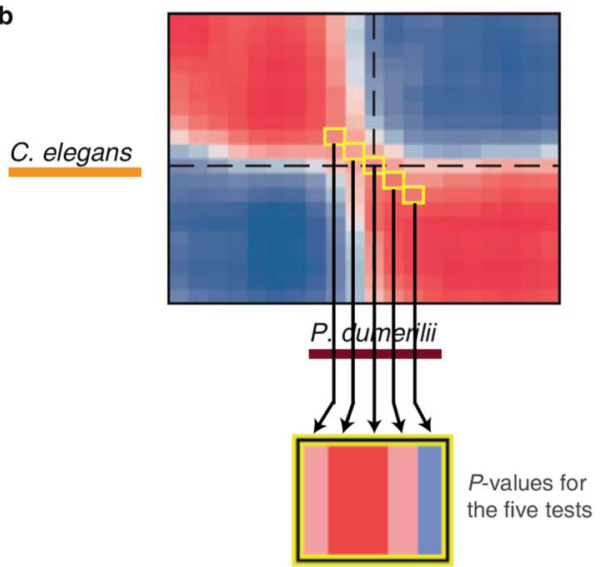

c
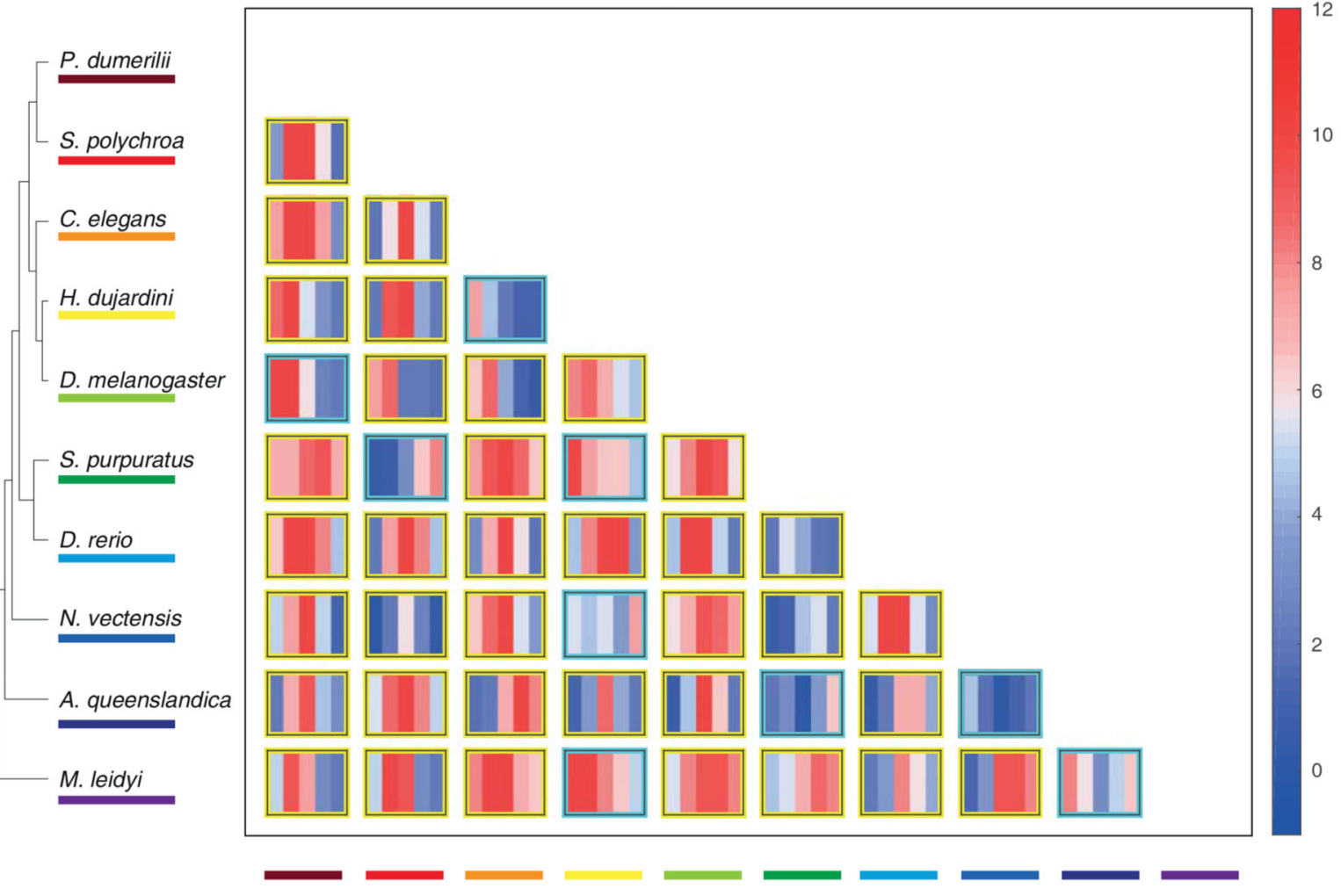

Extended Data Figure 3.

Testing the significance of the transition in the ortholog correlation matrices shown in Figure 3. a, schematic indicating the mid-developmental transition (orange), correlations among windows of the same phase (green), and correlations among windows of different phases (grey). b, The orange squares indicate statistical tests examined in c. c, For each pair of species a series of Kolmogorov-Smirnov tests are shown. Each test compares the intra-phase to the inter-phase correlations (a) for the mid-developmental transition and three windows before and after it (b). The yellow boxes indicate those species-comparisons where there is 
significant statistical evidence for the dual-phase pattern (higher significance for the middle tests).

a
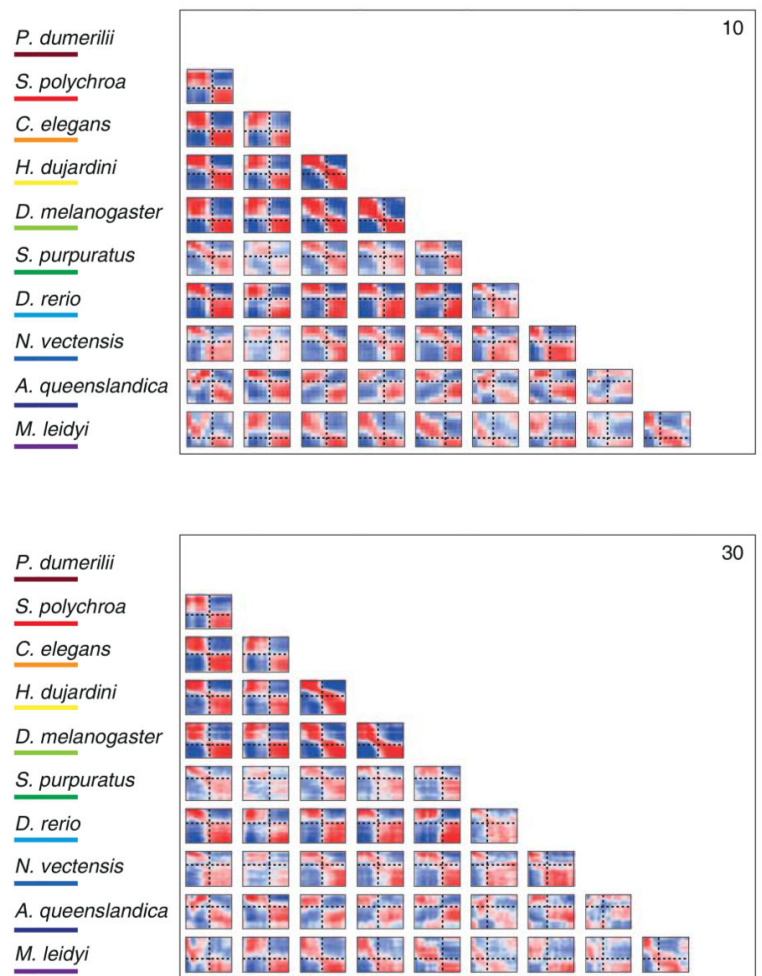
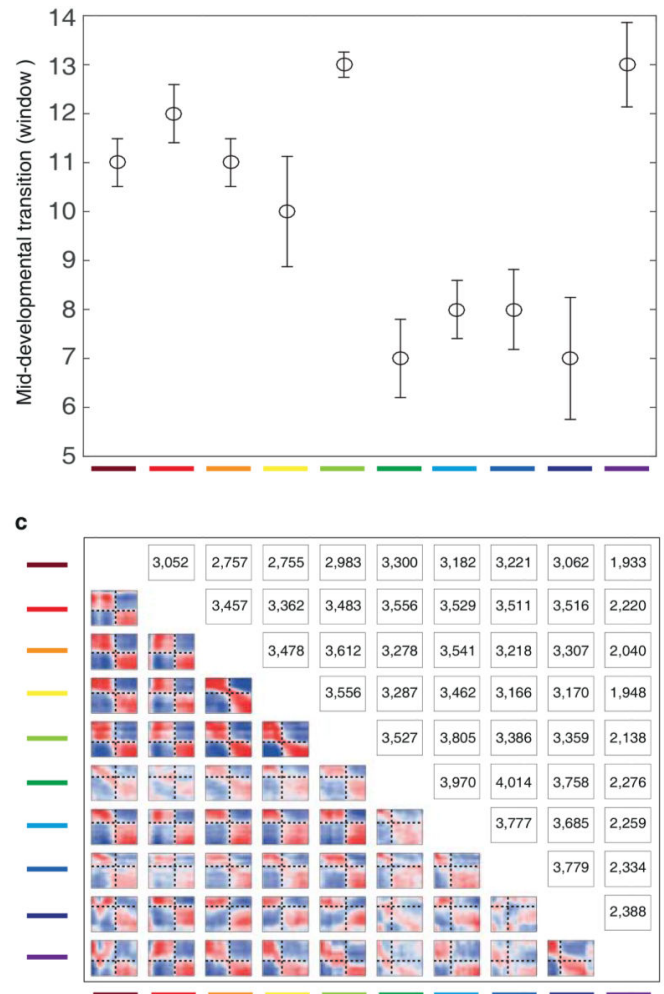

d

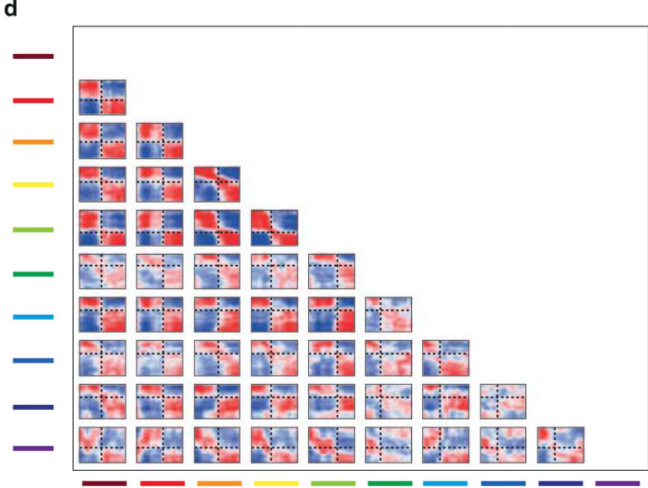

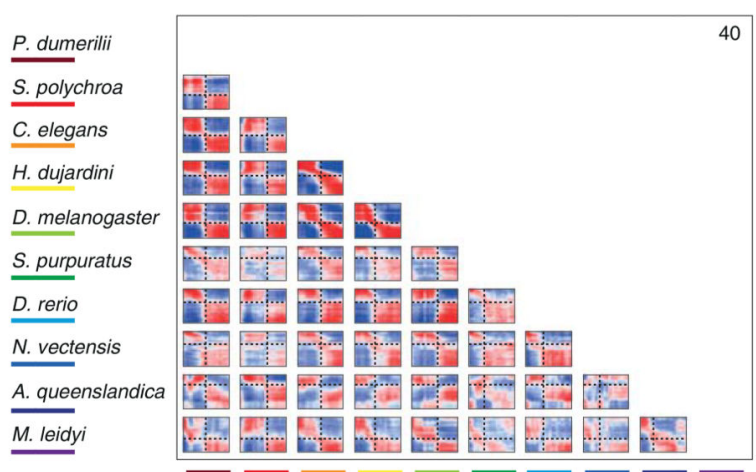

P. dumerilii

S. polychroa

C. elegans

H. dujardin

D. melanogaster

purpuratus

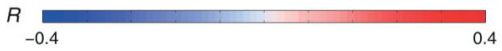

Extended Data Figure 4.

Robustness of Figure 3 analysis. a, The analysis was repeated using the indicated number of sliding windows. b, The analysis was repeated by randomly removing embryo transcriptomes. From each of the ten datasets, we removed $10 \%, 20 \%$, or $30 \%$ of the embryos and repeated the analysis using 20 windows. We repeated this five times and then 
re-identified the mid-developmental transition. The plot indicates the median and standard deviation of the detected mid-developmental transition windows across these trials. In all species, the median is identical to the mid-developmental transition identified by the complete dataset. c, d, Same as Figure 3, using (c) all detected orthologs for the pairwise comparisons (not limited to 1500 as in Figure 3, the number of examined orthologs are indicated above each plot), and (d) a set of 407 orthologs across all taxa.

a
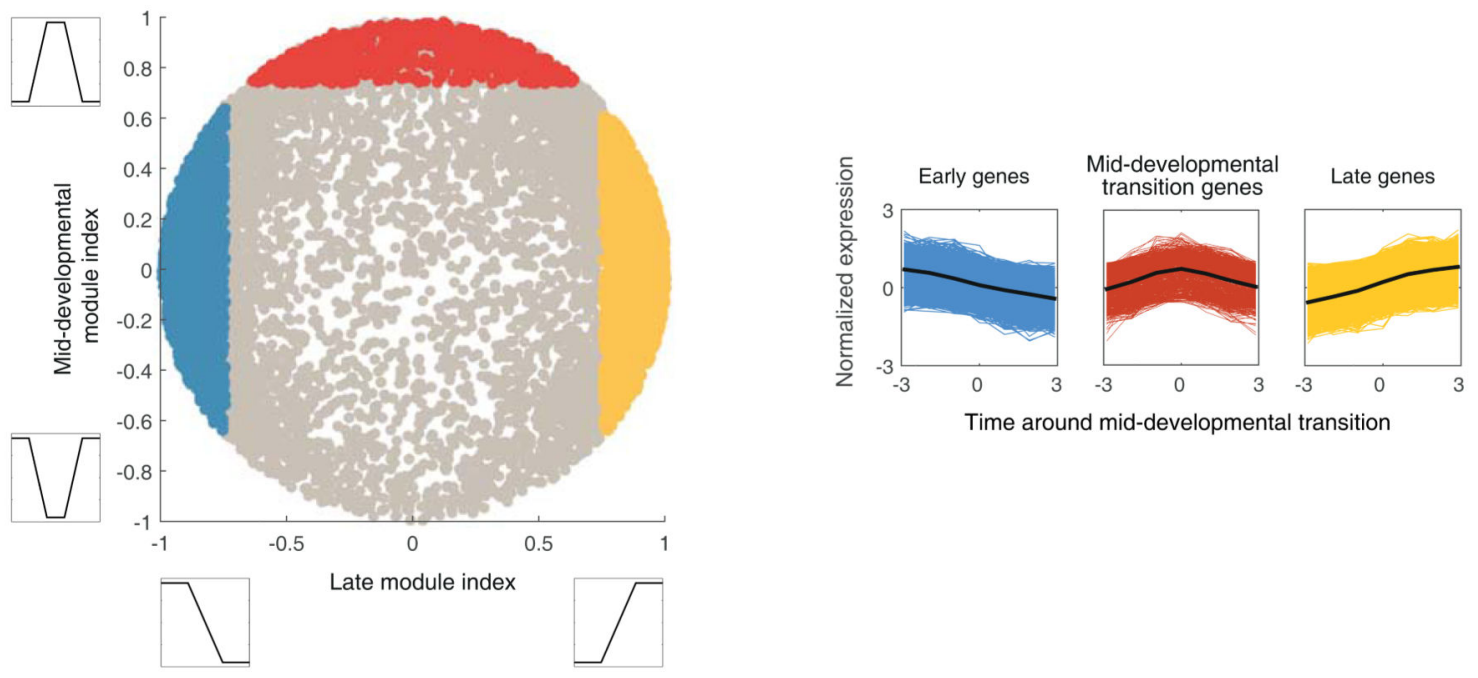

b

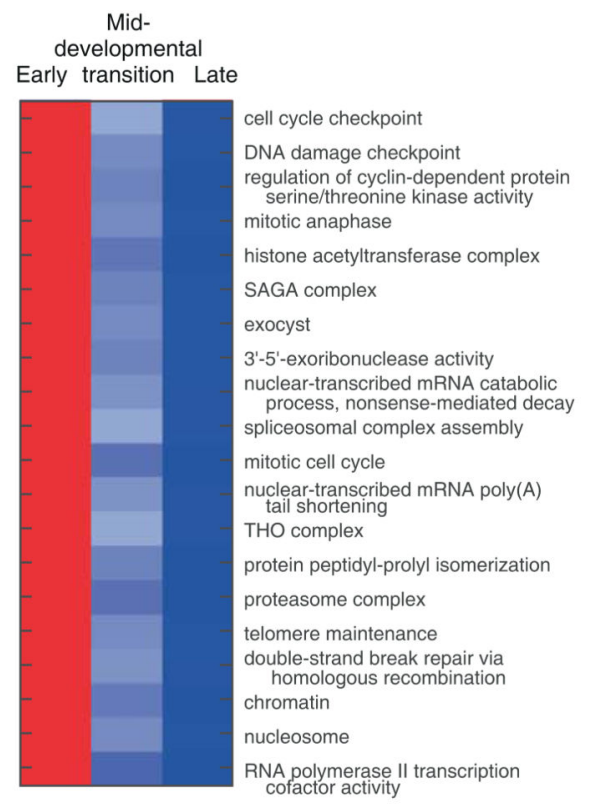

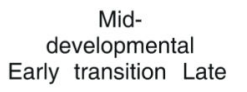

Early transition

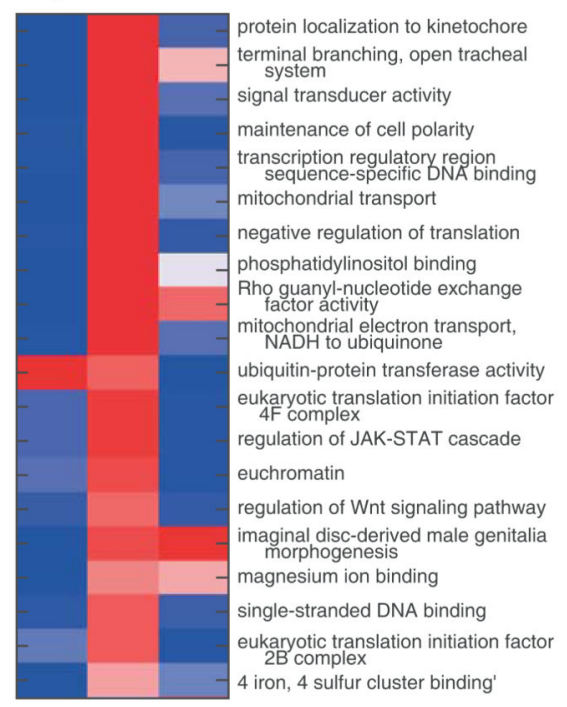

protein localization to kinetochore $\underset{\text { system }}{\text { s.m. }}$ signal transducer activity maintenance of cell polarity transcription regulatory region
sequence-specific DNA binding mitochondrial transport negative regulation of translation phosphatidylinositol binding Rho guanyl-nucleotide exchange mitochondrial electron transport, ubiquitin-protein transferase activity eukaryotic translation initiation factor regulation of JAK-STAT cascade euchromatin

regulation of Wnt signaling pathway maginal disc-derived male genitalia magnesium ion binding

single-stranded DNA binding eukaryotic translation initiation factor 4 iron, 4 sulfur cluster binding'

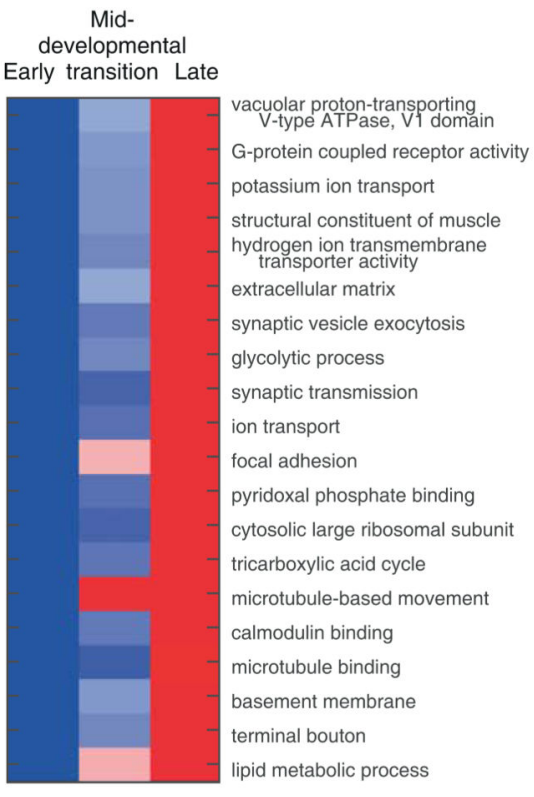

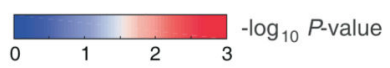

Extended Data Figure 5. 
a, A landscape showing for each gene (circle) the correlation with an idealized 'late module profile' (x-axis) and with a 'transition profile' (y-axis). The idealized profiles used to compute correlations are shown in the insets. Spots correspond to $C$. elegans genes. They are colored according to the assigned sets: early module (blue), mid-developmental transition (red), and late module (yellow).b, Gene Ontology (GO) enrichment for the early phase, transition, and late phase gene sets. The gene sets were defined by integrating expression from all ten species. 'RNA polymerase II ...' is short for 'RNA polymerase II core promoter sequence-specific DNA binding transcription factor activity involved in preinitiation complex assembly'. The legend indicates the assigned sets: early phase (blue), middevelopmental transition (red), and late phases (yellow). The profiles are of length nine since we examined four windows before and after the mid-developmental transition 

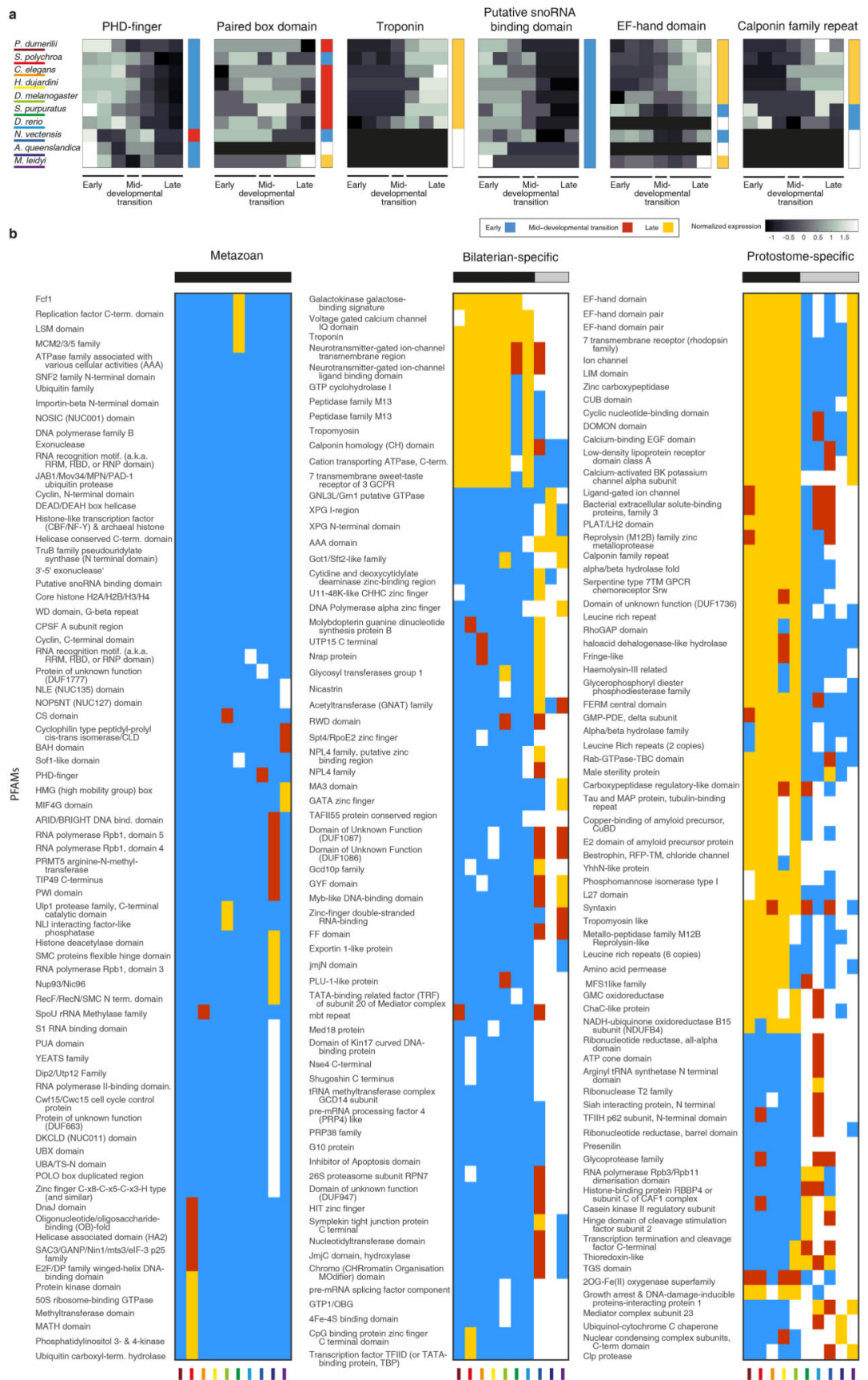

\section{Extended Data Figure 6.}

PFAM enrichment and conservation across phyla. For each of 5,746 PFAM protein domains, we computed an expression signature based upon the fraction of its genes expressed at each stage throughout development across each of the ten species. As an example of this approach, a shows the signatures for six PFAMs, indicating the normalized fraction of genes in that group expressed at the time-points surrounding the transition for each species. The profiles are centered according to the mid-developmental transition as defined in Figure 3, examining four windows before and after it. The gray-scale indicates the fraction of genes 
expressed in each window. We attributed a phase of expression for each PFAM in each species, as we have for individual genes. To the right of each PFAM signature is the annotated phase; early (blue), mid-developmental transition (red), or late (yellow). We then computed the degree to which the temporal expression across phyla matches a coherent phase expression in three groupings: metazoan, bilaterian, and protostomes. b, metazoan (all ten species), bilaterian (all except the cnidarian, sponge, and ctenophore), and protostome (nematode, arthropod, tardigrade, annelid, and platyhelminth). To identify PFAMs in the metazoan group, we queried for PFAMs whose signature contains the same times of expression across the species. The metazoan-coherent PFAMs were nearly exclusively expressed in the early phase, suggesting stronger evolutionary constraints on this phase. A similar pattern was also observed for PFAMs with coherent expression in the seven bilaterian species in our dataset. From this analysis, we conclude that bilaterians and protostomes each possess unique suites of innovations that are reflected in these shared phase-specific PFAM enrichments. Interestingly, protostome-coherent PFAMs are biased toward the late phases, possibly related to common differentiation processes operating in these taxa. 


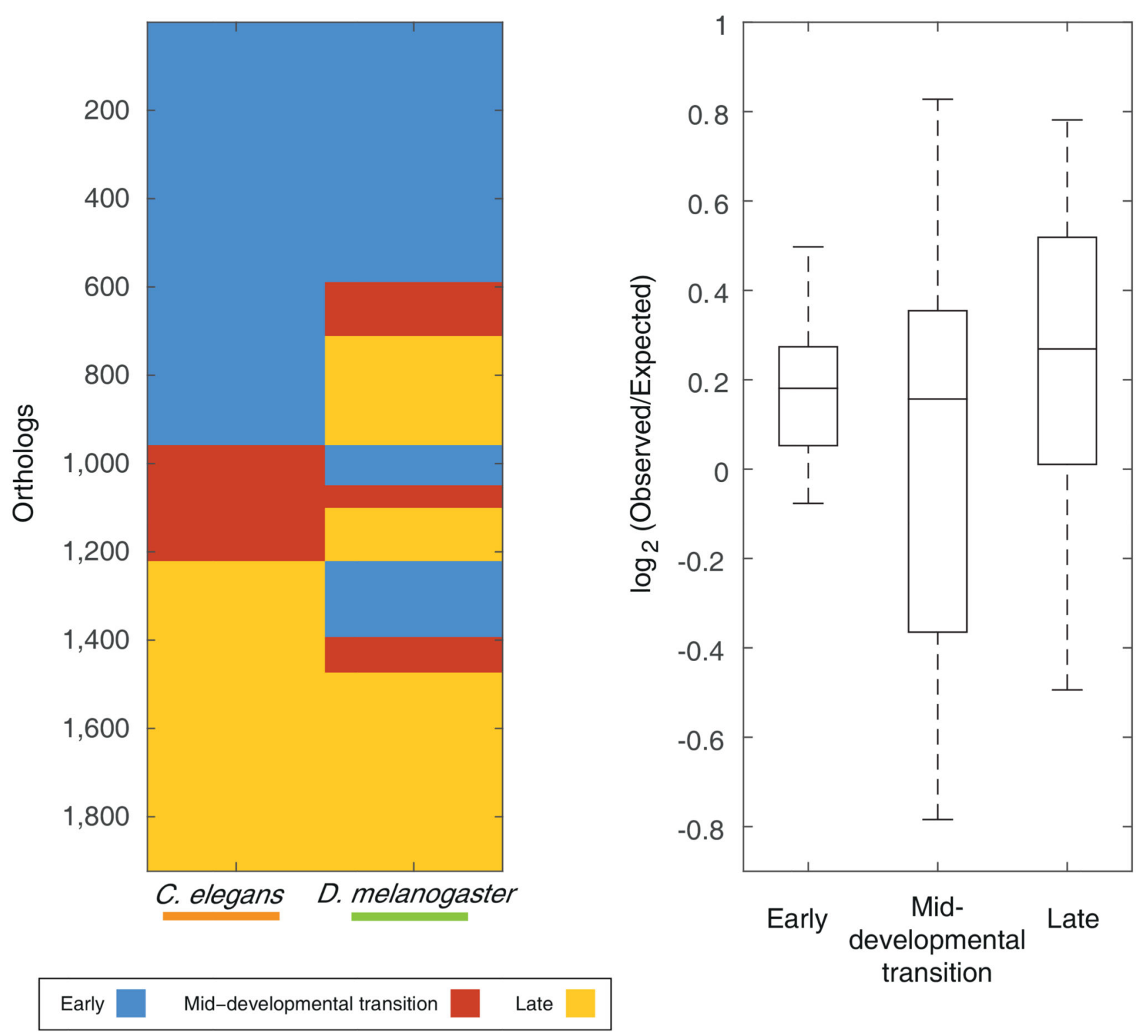

Extended Data Figure 7.

Same as Figure $4 \mathrm{c}, \mathrm{d}$ for PFAM analysis. The degree of conservation of early, transition, and late phase annotation of PFAMs was computed across species. A similar pattern was observed as that for orthologs (Figure 4c,d). 


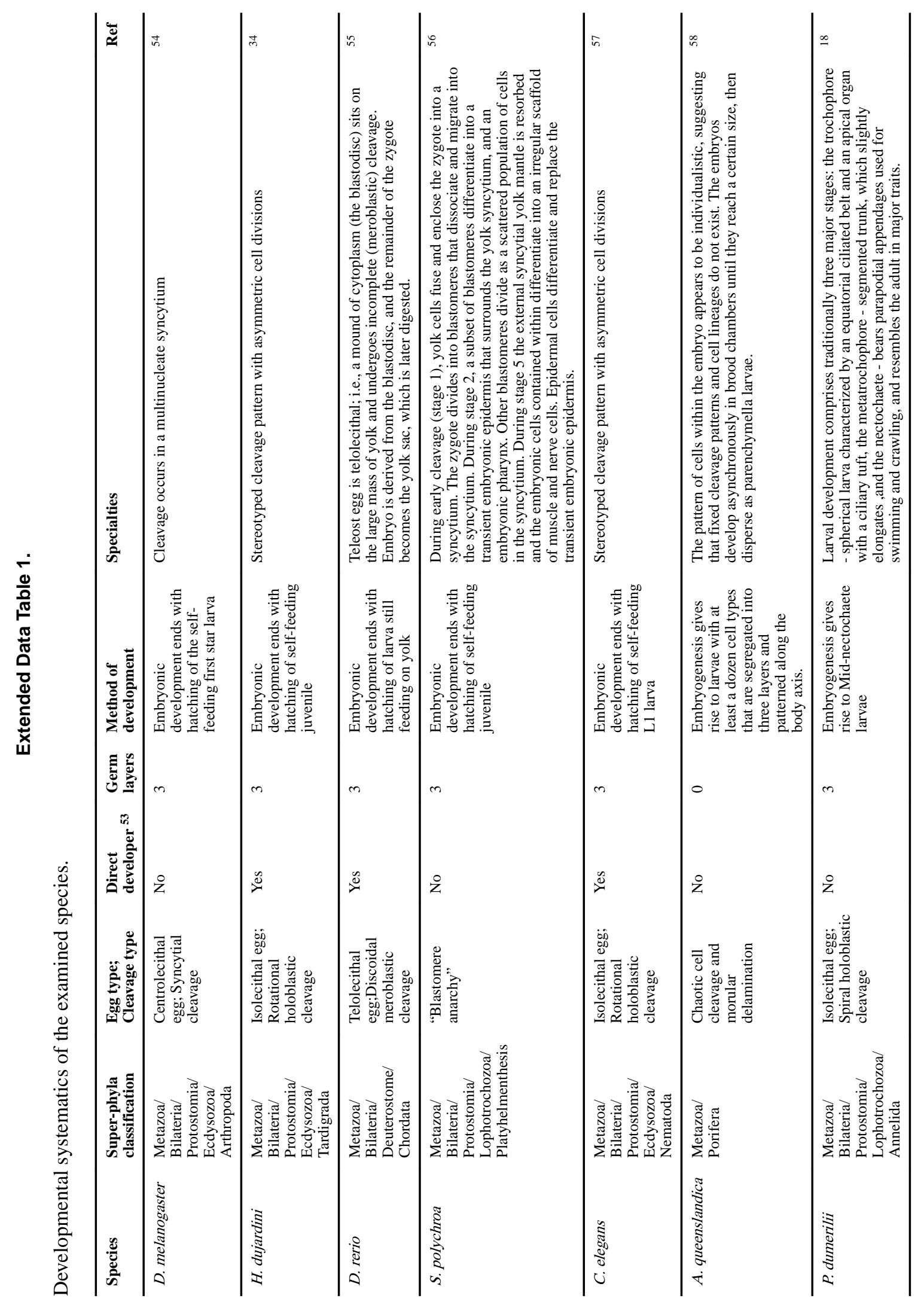

Nature. Author manuscript; available in PMC 2016 April 04. 


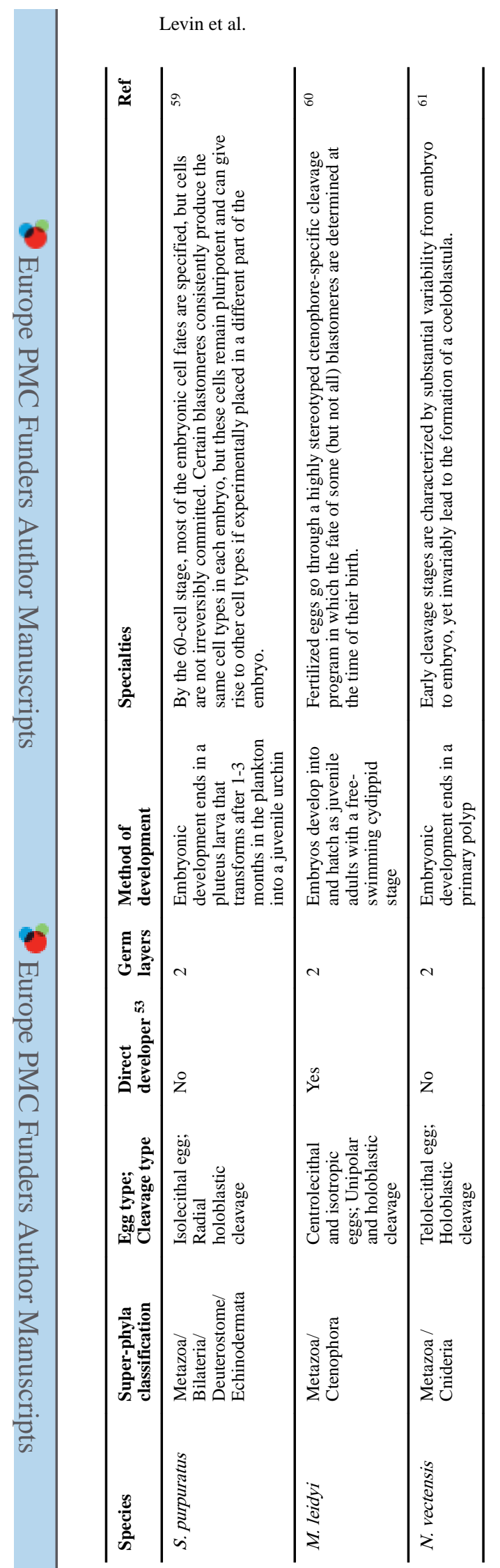

Nature. Author manuscript; available in PMC 2016 April 04. 


\section{Extended Data Table 2.}

Basic statistics of the expression data.

\begin{tabular}{lccccccc}
\hline Species & $\begin{array}{c}\text { Average } \\
\text { counted reads } \\
\text { (genes) }\end{array}$ & $\begin{array}{c}\text { Average } \\
\text { counted } \\
\text { reads (ERCC) }\end{array}$ & Genes & $\begin{array}{c}\text { Counted } \\
\text { genes }\end{array}$ & $\begin{array}{c}\text { Dynamically } \\
\text { expressed } \\
\text { genes }\end{array}$ & $\begin{array}{c}\text { Collected } \\
\text { samples }\end{array}$ & $\begin{array}{c}\text { Collected } \\
\text { samples }\end{array}$ \\
\hline P. dumerilii & 852,212 & 88,165 & 10,908 & 10,906 & 9,832 & 97 & 80 \\
\hline S. polychroa & 781,744 & 37,837 & 15,124 & 15,124 & 13,207 & 132 & 58 \\
\hline C. elegans & $1,364,849$ & 4,626 & 20,687 & 18,553 & 10,479 & 139 & 81 \\
\hline H. dujardini & $1,498,810$ & 199,916 & 12,746 & 12,744 & 11,464 & 126 & 62 \\
\hline D. melanogaster & $1,784,414$ & 90,767 & 15,682 & 13,842 & 8,391 & 91 & 77 \\
\hline S. purpuratus & 462,396 & 141,587 & 21,092 & 17,443 & 11,579 & 87 & 57 \\
\hline D. rerio & $2,010,010$ & 32,058 & 32,433 & 22,790 & 10,198 & 106 & 88 \\
\hline N. vectensis & $1,147,629$ & 176,166 & 40,499 & 37,272 & 16,106 & 123 & 83 \\
\hline A. queenslandica & $1,783,845$ & 45,455 & 44,719 & 35,922 & 10,053 & 63 & 51 \\
\hline M. leidyi & $1,583,798$ & 6,111 & 16,548 & 14,664 & 6,142 & 60 & 53 \\
\hline
\end{tabular}

Extended Data Table 3.

Assemblies for CEL-Seq mapping and their BUSCO completeness ${ }^{31}$

\begin{tabular}{|c|c|c|}
\hline Species & Assembly used for mapping & BUSCO \\
\hline P. dumerilii & Pdum_transcriptome_v1 (De Novo - NCBI BioProject PRJNA271451) & $57 \%$ \\
\hline S. polychroa & Spol_transcriptome_v1 (De Novo - NCBI BioProject PRJNA271420) & $70 \%$ \\
\hline C. elegans & WS230 (WormBase) & $90 \%$ \\
\hline H. dujardini & Hduj_transcriptome_v1 (De Novo - NCBI BioProject PRJNA271450) & $71 \%$ \\
\hline D. melanogaster & BDGP5 (Ensembl) & $99 \%$ \\
\hline S. purpuratus & WHL22 transcriptome ${ }^{63,64}$ & $78 \%$ \\
\hline D. rerio & Zv9 (Ensembl) & $96 \%$ \\
\hline$N$. vectensis & NvT1 (Stellabae) ${ }^{65}$ & $78 \%$ \\
\hline A. queenslandica & Aqu2. $1^{66}$ & $83 \%$ \\
\hline M. leidyi & M1Scaffold0911,67 & $70 \%$ \\
\hline
\end{tabular}

\section{Supplementary Material}

Refer to Web version on PubMed Central for supplementary material.

\section{Acknowledgements}

We thank Matt Rockman and Ben de Bivort for helpful discussions. We also thank Uri Gat, Adi Salzberg, Smadar Ben Tabou De Leon, Mark Blaxter, Georgios Koutsovoulos, Sarah Mansour, and Bob Goldstein for materials and reagents. We are grateful for the constructive comments of the anonymous referees. We thank the Technion Genome Center for technical assistance and the Radcliffe Institute for Advanced Studies at Harvard University for hosting the analysis phase. This work was supported by a European Research Council grant (EvoDevoPaths), the EMBO Young Investigator Program, and a grant from the Australian Research Council. 


\section{Author Contributions}

M.L., T.H., and I.Y. conceived and designed the project. M.L. led the collection of samples with help from N.N., D.S., N.M., S.K. and A.J.V. The processing of the samples for CELSeq was carried out by M.L., T.H., N.M., S.K., and N.S. De novo transcriptome assembly was carried out by L.A. with assistance from I.Y., E.W., J.F.R., and S.Y.L. Processing of CEL-Seq reads and initial bioinformatics was performed by L.A. with significant help from M.F., E.W., E.K., D.H.S, and S.L.F.V. The data was analyzed by I.Y. and L.A. I.Y. coordinated the interpretation of the data with significant help from B.M.D.; L.A., S.M.D., T.H., A.G.C., D.A., J.F.R., M.Q.M., K.Y., and J.C.R. also contributed to the interpretation of the data. I.Y. wrote the manuscript on which the authors commented.

\section{References}

1. Valentine, JW. On the Origin of Phyla. University of Chicago Press; 2004.

2. Haeckel, E. The Evolution of Man. Vol. 1. C. K. Paul \& Company; 1879.

3. Darwin, C. On the Origin of Species by Means of Natural Selection, Or, The Preservation of Favoured Races in the Struggle for Life. J. Murray; 1859.

4. Gould, SJ. Ontogeny and Phylogeny. Harvard University Press; 1977.

5. Abzhanov A. Von Baer's law for the ages: Lost and found principles of developmental evolution. Trends in Genetics. 2013; 29:712-722. [PubMed: 24120296]

6. Kalinka AT, et al. Gene expression divergence recapitulates the developmental hourglass model. Nature. 2010; 468:811-4. [PubMed: 21150996]

7. Levin M, Hashimshony T, Wagner F, Yanai I. Developmental milestones punctuate gene expression in the Caenorhabditis embryo. Dev. Cell. 2012; 22:1101-8. [PubMed: 22560298]

8. Domazet-Lošo T, Tautz D. A phylogenetically based transcriptome age index mirrors ontogenetic divergence patterns. Nature. 2010; 468:815-8. [PubMed: 21150997]

9. Irie N, Kuratani S. Comparative transcriptome analysis reveals vertebrate phylotypic period during organogenesis. Nat. Commun. 2011; 2:248. [PubMed: 21427719]

10. Scholtz, G. Evolutionary Developmental Biology of Crustacea. Crustacean Issues 15. A. A. Balkema; 2004. p. 3-16.

11. Ryan JF, et al. The genome of the ctenophore Mnemiopsis leidyi and its implications for cell type evolution. Science. 2013; 342:1242592. [PubMed: 24337300]

12. Hashimshony T, Wagner F, Sher N, Yanai I. CEL-Seq: single-cell RNA-Seq by multiplexed linear amplification. Cell Rep. 2012; 2:666-73. [PubMed: 22939981]

13. Anavy L, et al. BLIND ordering of large-scale transcriptomic developmental timecourses. Development. 2014; 141:1161-6. [PubMed: 24504336]

14. Davidson, EH. The Regulatory Genome: Gene Regulatory Networks In Development And Evolution. Academic Press; 2006.

15. Ballard WW. Morphogenetic movements and fate maps of vertebrates. Am. Zool. 1981; 21:391399.

16. Patel NH. Developmental evolution: insights from studies of insect segmentation. Science. 1994; 266:581-90. [PubMed: 7939712]

17. Sander, K. Development and Evolution. Goodwin, BC.; Holder, N.; Wylie, CC., editors. Cambridge University Press; 1983. p. 137-160.

18. Fischer AH, Henrich T, Arendt D. The normal development of Platynereis dumerilii (Nereididae, Annelida). Front. Zool. 2010; 7:31. [PubMed: 21192805]

19. Slack, JMW. Keywords and Concepts in Evolutionary Developmental Biology. Hall, BK.; Olson, WM., editors. Harvard University Press; 2003. p. 476

20. Cardona A, Hartenstein V, Romero R. Early embryogenesis of planaria: a cryptic larva feeding on maternal resources. Dev. Genes Evol. 2006; 216:667-81. [PubMed: 16932928] 
21. Martín-Durán JM, Egger B. Developmental diversity in free-living flatworms. Evodevo. 2012; 3:7. [PubMed: 22429930]

22. Adamska M, et al. The evolutionary origin of hedgehog proteins. Current Biology. 2007; 17

23. Raff RA. The shape of life: genes, development, and the evolution of animal shape. 1996; II

24. Richardson MK. Vertebrate evolution: the developmental origins of adult variation. Bioessays. 1999; 21:604-13. [PubMed: 10472187]

25. Slack JM, Holland PW, Graham CF. The zootype and the phylotypic stage. Nature. 1993; 361:4902. [PubMed: 8094230]

26. Larroux C, et al. The NK homeobox gene cluster predates the origin of Hox genes. Curr. Biol. 2007; 17:706-10. [PubMed: 17379523]

27. Ryan JF, Pang K, Mullikin JC, Martindale MQ, Baxevanis AD. The homeodomain complement of the ctenophore Mnemiopsis leidyi suggests that Ctenophora and Porifera diverged prior to the ParaHoxozoa. Evodevo. 2010; 1:9. [PubMed: 20920347]

28. Hashimshony T, Feder M, Levin M, Hall BK, Yanai I. Spatiotemporal transcriptomics reveals the evolutionary history of the endoderm germ layer. Nature. 2014; 519:219-22. [PubMed: 25487147]

29. Hejnol A, et al. Assessing the root of bilaterian animals with scalable phylogenomic methods. Proc. Biol. Sci. 2009; 276:4261-70. [PubMed: 19759036]

30. Dunn CW, et al. Broad phylogenomic sampling improves resolution of the animal tree of life. Nature. 2008; 452:745-9. [PubMed: 18322464]

\section{Methods References}

31. Simão FA, Waterhouse RM, Ioannidis P, Kriventseva EV, Zdobnov EM. BUSCO: assessing genome assembly and annotation completeness with single-copy orthologs. Bioinformatics. 2015 doi:10.1093/bioinformatics/btv351.

32. Martín-Durán JM, Duocastella M, Serra P, Romero R. New method to deliver exogenous material into developing planarian embryos. J. Exp. Zool. B. Mol. Dev. Evol. 2008; 310:668-81. [PubMed: 18942102]

33. Martín-Durán JM, Amaya E, Romero R. Germ layer specification and axial patterning in the embryonic development of the freshwater planarian Schmidtea polychroa. Dev. Biol. 2010; 340:145-58. [PubMed: 20100474]

34. Gabriel WN, et al. The tardigrade Hypsibius dujardini, a new model for studying the evolution of development. Dev. Biol. 2007; 312:545-59. [PubMed: 17996863]

35. Kiehart DP, Crawford JM, Montague RA. Collection, dechorionation, and preparation of Drosophila embryos for quantitative microinjection. CSH Protoc. 2007; 2007 pdb.prot4717.

36. Essential Zebrafish Methods: Cell and Developmental Biology. Academic Press; 2009.

37. Fritzenwanker JH, Technau U. Induction of gametogenesis in the basal cnidarian Nematostella vectensis(Anthozoa). Dev. Genes Evol. 2002; 212:99-103. [PubMed: 11914942]

38. Pang K, Martindale MQ. Mnemiopsis leidyi Spawning and Embryo Collection. CSH Protoc. 2008; 2008 pdb.prot5085.

39. Joshi, N.; Fass, J. Sickle: A sliding-window, adaptive, quality-based trimming tool for FastQ files. 2011.

40. Vince, B. Scythe - A Bayesian adapter trimmer. 2011.

41. Gnerre S, et al. High-quality draft assemblies of mammalian genomes from massively parallel sequence data. Proc. Natl. Acad. Sci. U. S. A. 2011; 108:1513-8. [PubMed: 21187386]

42. Boothby TC, et al. Evidence for extensive horizontal gene transfer from the draft genome of a tardigrade. Proc. Natl. Acad. Sci. 2015; 112:201510461.

43. Koutsovoulos, G., et al. The genome of the tardigrade Hypsibius dujardini. Cold Spring Harbor Labs Journals; 2015. bioRxivdoi:10.1101/033464

44. Sullivan JC, Reitzel AM, Finnerty JR. Upgrades to StellaBase facilitate medical and genetic studies on the starlet sea anemone, Nematostella vectensis. Nucleic Acids Res. 2008; 36:D607-11.

[PubMed: 17982171] 
45. Hashimshony T, Wagner F, Sher N, Yanai I. CEL-Seq: single-cell RNA-Seq by multiplexed linear amplification. Cell Rep. 2012; 2:666-73. [PubMed: 22939981]

46. Baker SC, et al. The External RNA Controls Consortium: a progress report. Nat. Methods. 2005; 2:731-734. [PubMed: 16179916]

47. Langmead B, Salzberg SL. Fast gapped-read alignment with Bowtie 2. Nat. Methods. 2012; 9:357359. [PubMed: 22388286]

48. Anders S, Pyl PT, Huber W. HTSeq - A Python framework to work with high-throughput sequencing data. Bioinformatics. 2014 doi:10.1093/bioinformatics/btu638.

49. Haas BJ, et al. De novo transcript sequence reconstruction from RNA-seq using the Trinity platform for reference generation and analysis. Nat. Protoc. 2013; 8:1494-1512. [PubMed: 23845962]

50. Altschul SF, Gish W, Miller W, Myers EW, Lipman DJ. Basic local alignment search tool. J. Mol. Biol. 1990; 215:403-10. [PubMed: 2231712]

51. Punta M, et al. The Pfam protein families database. Nucleic Acids Res. 2012; 40:D290-301. [PubMed: 22127870]

52. Finn RD, Clements J, Eddy SR. HMMER web server: interactive sequence similarity searching. Nucleic Acids Res. 2011; 39:W29-37. [PubMed: 21593126]

53. Li L, Stoeckert CJ, Roos DS. OrthoMCL: identification of ortholog groups for eukaryotic genomes. Genome Res. 2003; 13:2178-89. [PubMed: 12952885] 
a

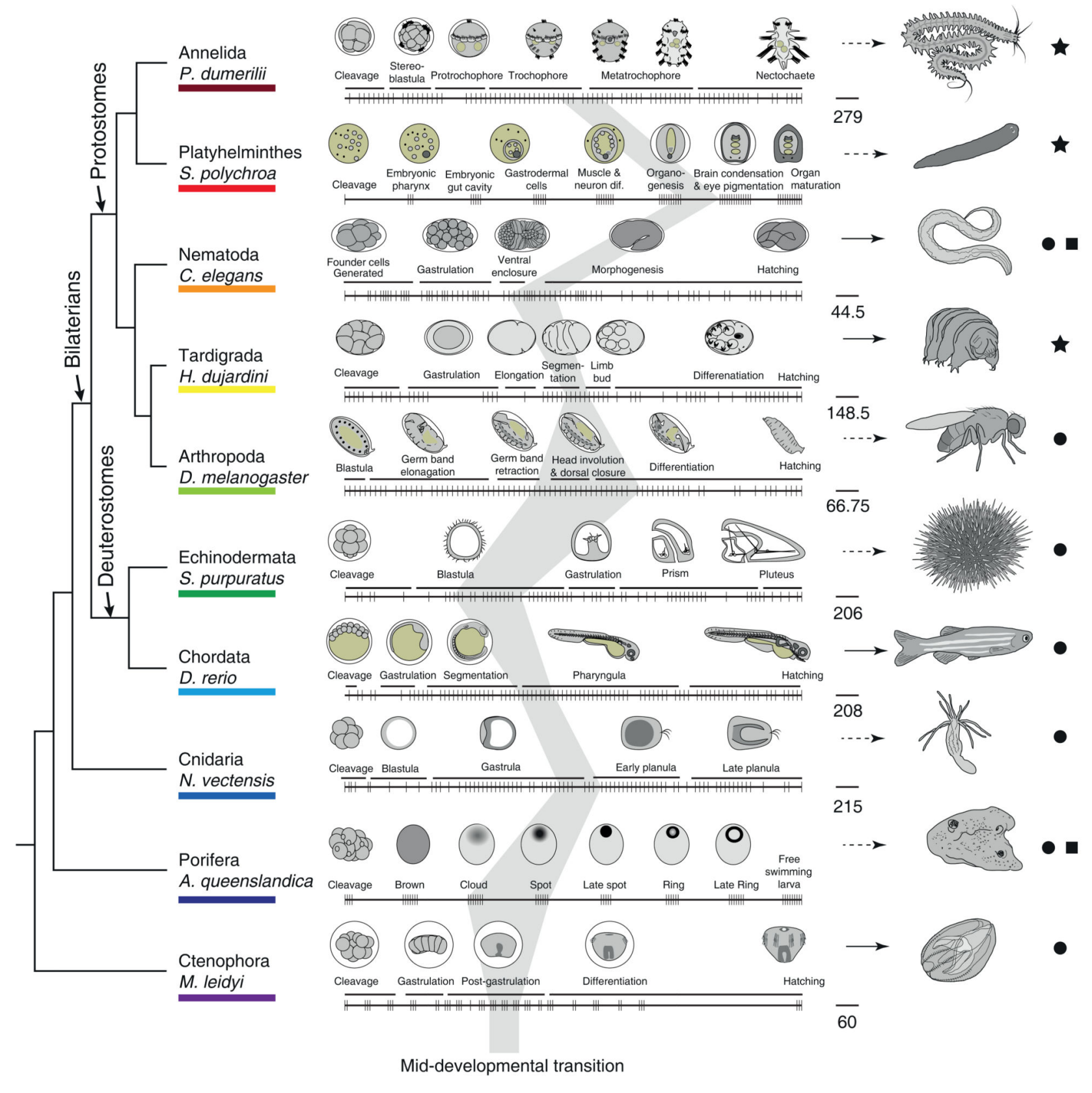

Figure 1. Comparing development across ten phyla using CEL-Seq

a, A phylogeny of the examined species based on recent work ${ }^{11,29,30}$. b, A representation of the times (notches) at which individual embryos were collected. Drawings of embryos at the indicated representative stages are shown above the collection time-course (on the right, time-scale in minutes). The dark grey shading indicates the mid-developmental transitions. Stars, species where the developmental time-course is mapped to a mixed-stage transcriptome reported here; Circles, mapped to the previously published genomes; Squares, 
previously published time-courses ${ }^{13,28}$. Arrows indicate direct (solid) and indirect (dashed) developers. 


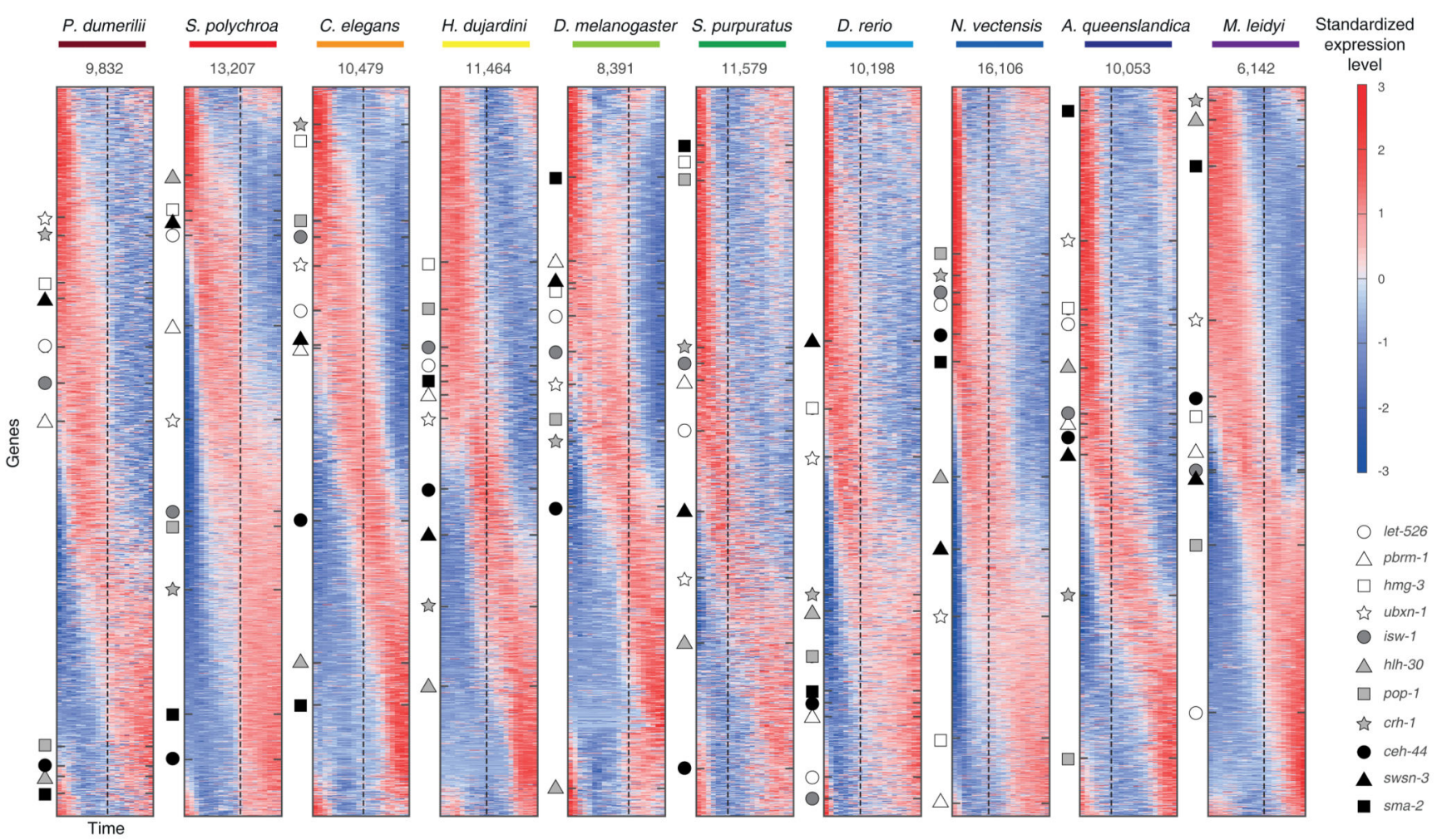

Figure 2. Dynamic embryonic expression throughout the animal kingdom Sorted standardized temporal gene expression profiles for each species. Genes of eleven orthologous groups are indicated by the markers along with the name of the $C$. elegans ortholog. The dashed lines indicate the timing of the mid-developmental transition. The number indicates expressed genes is each dataset. 


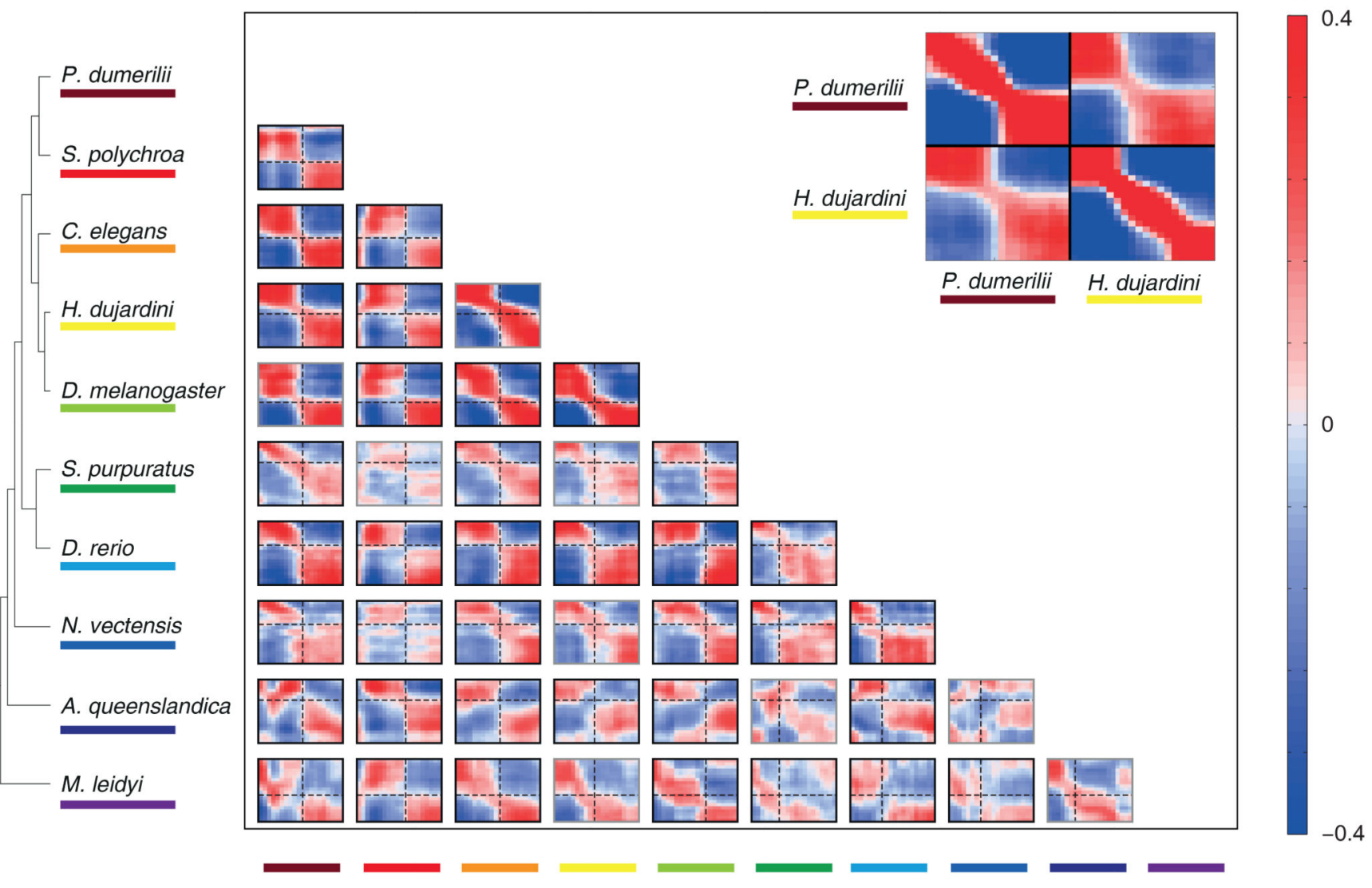

Figure 3. Cross-phyla comparison of developmental transcriptomes

Each heatmap shows the correlations between the developmental transcriptomes of a pair of species based upon analysis of 1,500 highly-expressed orthologs (similar results are observed for other sets of orthologs, Extended Data Figure 4c,d). Dashed lines indicate the mid-developmental transitions. The grey box outlines indicate species-pairs in which the transition is not significant (Extended Data Figure 3). The inset shows the comparison for $H$. dujardini and $P$. dumerill, showing also the self-correlations. 
a
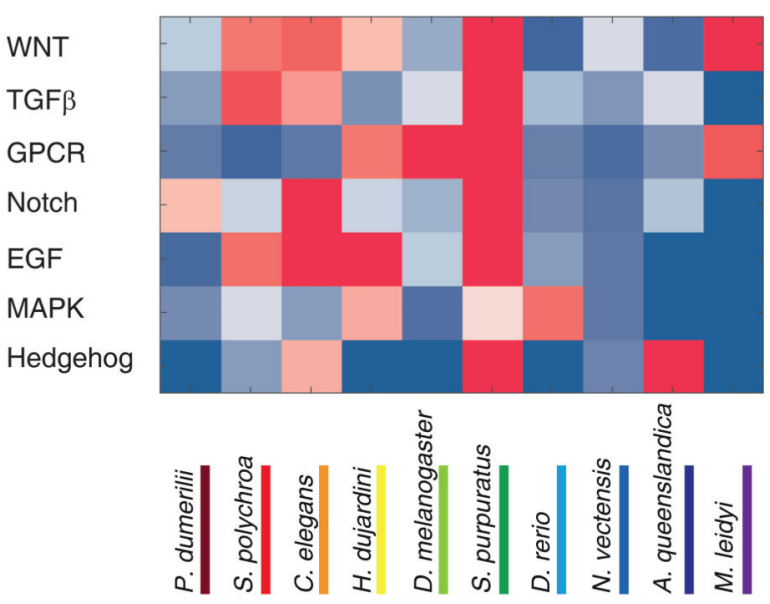

C

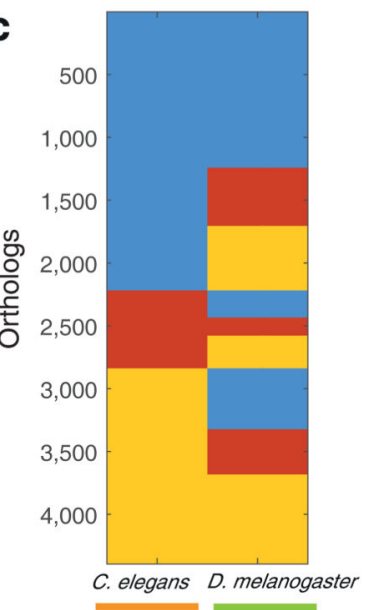

Early

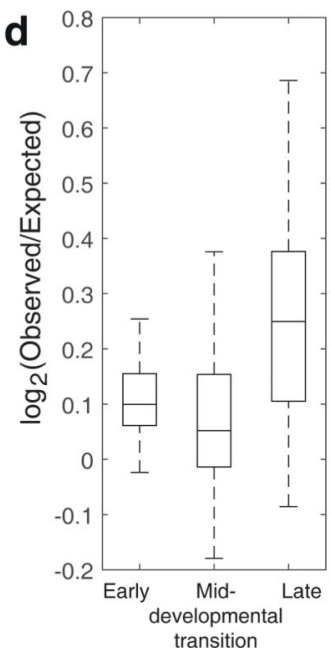

b

NHR

NR zinc

GATA

Multi-zinc

Homeodomain

$\beta \mathrm{HLH}$

$\beta$ blP

T-box

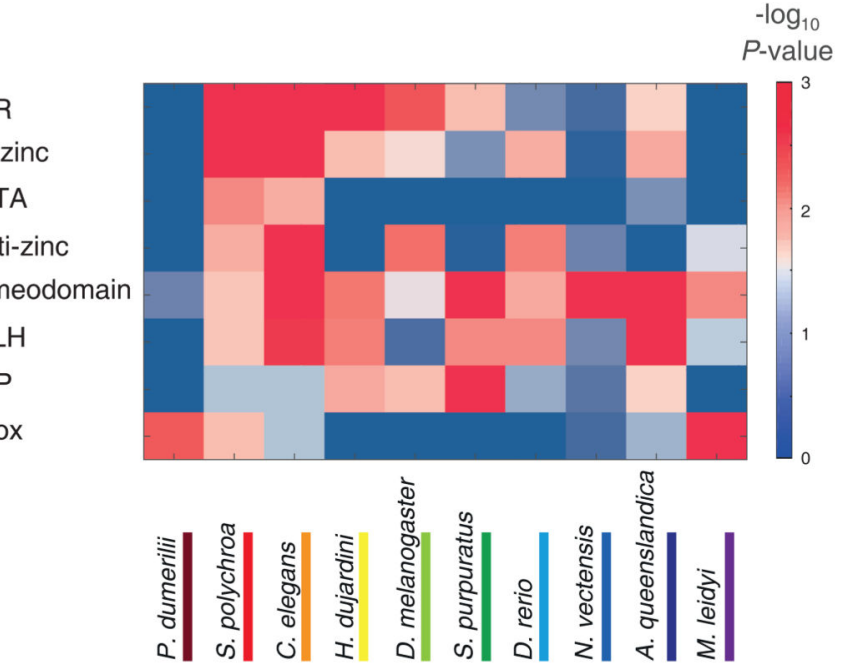

e

Hourglass model for within phylum similarity

Inverse hourglass model for cross-phyla similarity

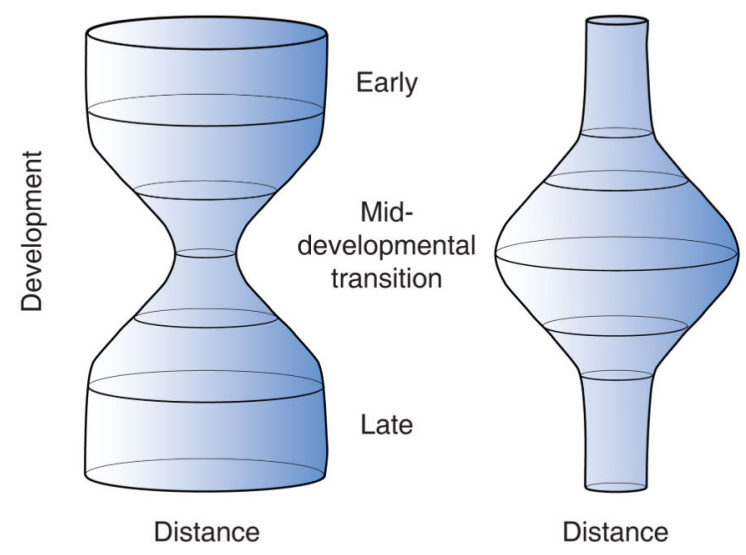

Figure 4. An inverse hourglass model for animal evolution

a,b, Functional enrichments for expression of seven signaling pathways (a) and seven transcription factor families (b) during the mid-developmental transitions. c, Comparison of the ortholog temporal associations between $C$. elegans and D. melanogaster. d, Summary of the 45 pairwise species comparisons of ortholog temporal associations. e, Inverse hourglass model for the origin of phyla compared with the hourglass model for within phylum evolution. 